Review

\title{
Experimental Methods to Simulate and Evaluate Postsurgical Peripheral Nerve Scarring
}

\author{
Alessandro Crosio ${ }^{1, \dagger}{ }^{,}$Giulia Ronchi ${ }^{2, \dagger}$, Benedetta Elena Fornasari $\left.{ }^{2}{ }^{(}\right)$, Simonetta Odella ${ }^{1}(\mathbb{D}$, \\ Stefania Raimondo ${ }^{2, *(\mathbb{D})}$ and Pierluigi Tos 1 \\ 1 UO Microchirurgia e Chirurgia della Mano, Ospedale Gaetano Pini, Piazza Andrea Ferrari 1, \\ 20122 Milano, Italy; Alessandro.Crosio@asst-pini-cto.it (A.C.); simonettaodella@gmail.com (S.O.); \\ Pierluigi.Tos@asst-pini-cto.it (P.T.) \\ 2 Department of Clinical and Biological Sciences, Neuroscience Institute of the "Cavalieri Ottolenghi" \\ Foundation (NICO), University of Turin, Regione Gonzole 10, 10043 Orbassano, Italy; \\ giulia.ronchi@unito.it (G.R.); benedettaelena.fornasari@unito.it (B.E.F.) \\ * Correspondence: stefania.raimondo@unito.it; Tel.: +39-011-670-5433 \\ + A.C. and G.R. contributed equally to this work.
}

Citation: Crosio, A.; Ronchi, G.; Fornasari, B.E.; Odella, S.; Raimondo, S.; Tos, P. Experimental Methods to Simulate and Evaluate Postsurgical Peripheral Nerve Scarring. J. Clin. Med. 2021, 10, 1613. https:// doi.org/10.3390/jcm10081613

Academic Editor: Lucas Prantl

Received: 27 January 2021

Accepted: 7 April 2021

Published: 10 April 2021

Publisher's Note: MDPI stays neutral with regard to jurisdictional claims in published maps and institutional affiliations.

Copyright: (c) 2021 by the authors. Licensee MDPI, Basel, Switzerland. This article is an open access article distributed under the terms and conditions of the Creative Commons Attribution (CC BY) license (https:// creativecommons.org/licenses/by/ $4.0 /)$.

\begin{abstract}
As a consequence of trauma or surgical interventions on peripheral nerves, scar tissue can form, interfering with the capacity of the nerve to regenerate properly. Scar tissue may also lead to traction neuropathies, with functional dysfunction and pain for the patient. The search for effective antiadhesion products to prevent scar tissue formation has, therefore, become an important clinical challenge. In this review, we perform extensive research on the PubMed database, retrieving experimental papers on the prevention of peripheral nerve scarring. Different parameters have been considered and discussed, including the animal and nerve models used and the experimental methods employed to simulate and evaluate scar formation. An overview of the different types of antiadhesion devices and strategies investigated in experimental models is also provided. To successfully evaluate the efficacy of new antiscarring agents, it is necessary to have reliable animal models mimicking the complications of peripheral nerve scarring and also standard and quantitative parameters to evaluate perineural scars. So far, there are no standardized methods used in experimental research, and it is, therefore, difficult to compare the results of the different antiadhesion devices.
\end{abstract}

Keywords: scar tissue; peripheral nerve regeneration; antiadhesion devices; animal models

\section{Introduction}

Scar tissue around the nerve can arise as a consequence of traumatic injuries and surgical procedures on peripheral nerves. This condition easily worsens the capacity of the peripheral nerve to regenerate and can give rise to traction neuropathies. Nerve tethering in the surgical scar is still the main cause of symptoms related to perineural scarring [1].

Traction neuropathies can be the consequence of elective procedures, including nerve decompression, primary nerve repair, and so on. For instance, $7-20 \%$ of patients subjected to primary median nerve release report pain and symptom recurrence [2,3]. Thus, peripheral nerve injuries compromise the quality of life of affected people, with a consequent important socioeconomic impact [4-6].

This condition is difficult to manage; according to different reports, compression symptoms persist after $40-90 \%$ of revision procedures, and $20 \%$ of patients actually require a third operation [7]. Moreover, $5 \%$ of nerve sutures have been estimated to induce a pain syndrome [8].

A primary role in this pathological condition has been attributed to the formation of scar tissue around the injured nerve. In particular, extrinsic nerve scarring occurs at the periphery of the epineurium, while intrinsic nerve scarring occurs within the nerve and can surround neural structures at all levels (both perineurium and endoneurium) [9]. To 
overcome scar tissue formation, a lot of different antiadhesion devices have been tested, developed, and introduced in clinical practice.

Since the late 1990s, researchers have tried to develop experimental models to investigate the efficacy of different antiadhesion devices. Several types of devices have been tested so far, but the methods employed to produce, simulate, and evaluate postsurgical scars are completely inhomogeneous and not reproducible [10]. This makes it difficult to compare the efficacy of the different antiadherence strategies in order to optimize clinical treatment.

The aim of this review is to illustrate the different methods adopted in experimental research to simulate and evaluate postsurgical scars. Finally, an overview of the antiadhesion devices tested so far in preclinical research is also provided.

\section{Materials and Methods}

An extensive research on PubMed was performed, employing the following search string: "peripheral nerves AND fibrosis OR perineural scar OR scar neuropathy OR traction neuropathy AND prevention", limited to English, other animals, and between 1 January 1995 and 31 December 2020. Furthermore, the reference list of each article was screened in order to find any additional original articles. Selection by title, abstract, and text was then performed; a total of 60 papers were retrieved. We did our best to include all articles available; nevertheless, inadvertently, we could have missed some papers, and we apologize in advance to their authors.

\section{Scar Simulation: Animal and Nerve Choice}

The choice of an appropriate animal model for preclinical research depends on different factors, including the aim and duration of the study, the anatomy and physiology of the animal model, the size of the medical device that needs to be tested, and, of course, the similarity with human clinical characteristics of the disease/condition. Finally, the cost and care of the animal model (housing, feeding, and caring) can also be considered.

For the study of peripheral nerve scarring, the most employed animal model is the rat model, followed by mouse and rabbit used in a limited number of studies (see Tables 1-6). This can be due to the lower cost of rats compared to rabbits, the easier caregiving, and the faster scar formation in smaller rather than bigger animals. On the other hand, mice (and their nerves) are very small and, therefore, more difficult to manage. Additionally, different $\mathrm{rat} / \mathrm{rabbit} / \mathrm{mouse}$ strains have been used. No research with other animal species (sheep, pigs, monkeys, cats, or dogs) has been found, in contrast with studies on peripheral nerve regeneration, where sheep as an animal model is often used to test regeneration across long distances [11,12].

Moreover, the choice of nerve model can be guided by several factors, including nerve size and the surrounding tissues. Most researchers use the sciatic nerve because it can be easily dissected, and there are no surrounding vascular and nervous structures that can impair the efficacy of the study. Only a few papers have used other nerve models such as the ulnar [13,14], peroneal [15], and median nerves [16].

\section{Scar simulation: Experimental Methods to Induce Scar Formation}

The main aim of most of the research dealing with scar formation is to test the efficacy of antiadhesion devices. Only very few papers [17-21] are focused on the standardization of a scarring method without testing any antiadhesion device (Table 1). These papers are very important in this field because a shared, effective, reliable, and reproducible protocol to induce and evaluate the amount of scar tissue is needed to compare the efficacy of different antiadhesion devices; so far, this is not available [18,20-22]. 
Table 1. No antiscarring agents tested.

\begin{tabular}{|c|c|c|c|c|}
\hline Reference & $\begin{array}{l}\text { Method to Induce } \\
\text { Scar Formation }\end{array}$ & $\begin{array}{c}\text { Animal and Nerve } \\
\text { Model }\end{array}$ & Analyses & Results \\
\hline Lemke et al., 2017 [21] & $\begin{array}{l}\text { Application of } \\
\text { "glutaraldehyde glue" } \\
\text { on the nerve and } \\
\text { surrounding muscle } \\
\text { or scratching }\end{array}$ & $\begin{array}{l}\text { Female } \\
\text { Sprague-Dawley } \\
\text { Rat } \\
\text { Sciatic nerve }\end{array}$ & $\begin{array}{ll}\text { - } & \text { Functional evaluation } \\
\text { (CatWalk, SFI) (once } \\
\text { a week) } \\
\text { - } \quad \text { Gross evaluation } \\
\text { (Petersen's classification) } \\
\text { (3 weeks) } \\
\text { - } \quad \text { Histology on nerve and } \\
\text { surrounding tissue (H\&E, } \\
\text { Masson's trichrome and } \\
\text { Luxol fast blue, } \\
\text { chromotrop-aniline-blue) } \\
\text { (3 weeks) } \\
\text { IHC (2F11, S100, CD-68, } \\
\text { CD-3, CD-8) (3 weeks) } \\
\text { Electrophysiological } \\
\text { evaluation (3 weeks) }\end{array}$ & $\begin{array}{l}\text { Severe intra- and } \\
\text { perineural scarring, } \\
\text { vigorous nerve } \\
\text { inflammation and } \\
\text { nerve degeneration } \\
\text { and functional deficit. }\end{array}$ \\
\hline Crosio et al., 2014 [20] & Burning or scratching & $\begin{array}{l}\text { Male Mouse } \\
\text { Sciatic nerve }\end{array}$ & $\begin{array}{ll}\text { - } & \text { Biomechanical analysis } \\
\text { ( } 3 \text { weeks) } \\
\text { - } \quad \text { Histology on nerve and } \\
\text { surrounding tissue } \\
\text { (Picrosirius staining) } \\
\text { (3 weeks) }\end{array}$ & $\begin{array}{l}\text { Both methods } \\
\text { produced fibrotic } \\
\text { reactions with no } \\
\text { differences in } \\
\text { biomechanical results } \\
\text { between the two } \\
\text { methods; histology } \\
\text { showed a different } \\
\text { distribution pattern of } \\
\text { the scar tissue. }\end{array}$ \\
\hline $\begin{array}{l}\text { Okuhara Y et al., } \\
2014 \text { [19] }\end{array}$ & $\begin{array}{l}\text { Irradiation of the } \\
\text { nerve with } \\
\text { X-radiation }\end{array}$ & $\begin{array}{l}\text { Female } \\
\text { LEW/CrlCrlj Rat } \\
\text { Sciatic nerve }\end{array}$ & $\begin{array}{ll}\text { - } & \text { Functional evaluation } \\
\text { (SFI) }(4,8,12,16,20, \text { and } \\
\text { 24 weeks) } \\
\text { - } \quad \text { Electrophysiological } \\
\text { evaluation (24 weeks) } \\
\text { - } \quad \text { Gross evaluation } \\
\text { (qualitative) (24 weeks) } \\
\text { - } \quad \text { Histology on nerve and } \\
\text { surrounding tissue } \\
\text { (Masson's trichrome and } \\
\text { Toluidine Blue for } \\
\text { morphometric analysis) } \\
\text { (24 weeks) }\end{array}$ & $\begin{array}{l}\text { Scar formation around } \\
\text { the radiated nerve. No } \\
\text { differences in SFI } \\
\text { between groups, but } \\
\text { axonal degeneration in } \\
\text { the irradiated nerve. }\end{array}$ \\
\hline
\end{tabular}

- Functional evaluation (Toe Out Angle) (weekly up to 4 weeks)

Laceration, crush, mince, and burn of the surrounding muscles
Female Wistar Rat

Sciatic nerve
- Gross Evaluation (Petersen's classification) (1, 2, 3, 4 weeks)

- $\quad$ Histology on nerve and surrounding tissue (Masson's trichrome) (1, 2, 3, 4 weeks)
Scar tissue formation surrounding the sciatic nerve in gross examination and histological analysis; no differences in functional assessment compared to control. 
Table 1. Cont.

\begin{tabular}{|c|c|c|c|c|}
\hline Reference & $\begin{array}{l}\text { Method to Induce } \\
\text { Scar Formation }\end{array}$ & $\begin{array}{c}\text { Animal and Nerve } \\
\text { Model }\end{array}$ & Analyses & Results \\
\hline Abe et al., 2005 [17] & $\begin{array}{l}\text { Nerve bed } \\
\text { cauterization and } \\
\text { suturing the nerve in } \\
\text { place }\end{array}$ & $\begin{array}{l}\text { Male Japanese White } \\
\text { Rabbit } \\
\text { Sciatic nerve }\end{array}$ & $\begin{array}{ll}\text { - } & \begin{array}{l}\text { Gross evaluation } \\
\text { (classification based on }\end{array} \\
\text { Fontana's bands and } \\
\text { Sakurai's classification) } \\
(6,14,22,30, \text { or } \\
38 \text { weeks) } \\
\text { Electrophysiological } \\
\text { evaluation }(6,14,22,30, \\
\text { or } 38 \text { weeks) } \\
\text { Nerve fascicle blood } \\
\text { flow }(6,14,22,30, \text { or } \\
38 \text { weeks) } \\
\text { Histology on nerve and } \\
\text { surrounding tissue } \\
\text { (Toluidine Blue) }(6,14, \\
22,30, \text { or } 38 \text { weeks) }\end{array}$ & $\begin{array}{l}\text { Adhesion of peripheral } \\
\text { nerve to surrounding } \\
\text { tissues results in } \\
\text { fibrosis in the nerve. } \\
\text { Compound muscle } \\
\text { action potentials were } \\
\text { reduced in amplitude, } \\
\text { and blood flow was } \\
\text { significantly decreased } \\
\text { at adhesion sites in } \\
\text { Group IIb. }\end{array}$ \\
\hline
\end{tabular}

SFI (Sciatic functional index), IHC (Immunohistochemistry), 2F11 (antibody labelling neurofilament), S100 (antibody labelling Schwann cells), CD-68 (Cluster of Differentiation 68, antibody labelling macrophages), CD-3 (Cluster of Differentiation 3 , antibody labelling T cells), CD-8 (Cluster of Differentiation 8, antibody labelling cytotoxic T cells).

The methods used to induce scar formation are several (Figure 1), but two of these are more frequently used. The first one consists of a direct injury (mechanical, epiperineurectomy, suture and repair, thermal, chemical, or physical) applied to the nerve surface. The second one consists of inducing an injury in the surrounding muscular bed by means of electrocoagulation, triggering the process of fibrosis from the surrounding tissue. Some researchers have induced a global injury to the nerve and surrounding tissues by scratching both nerve and muscles with irradiation or chemical injuries. Furthermore, the envelopment of the nerve in a silastic tube in order to let the scar tissue rise has been proposed.

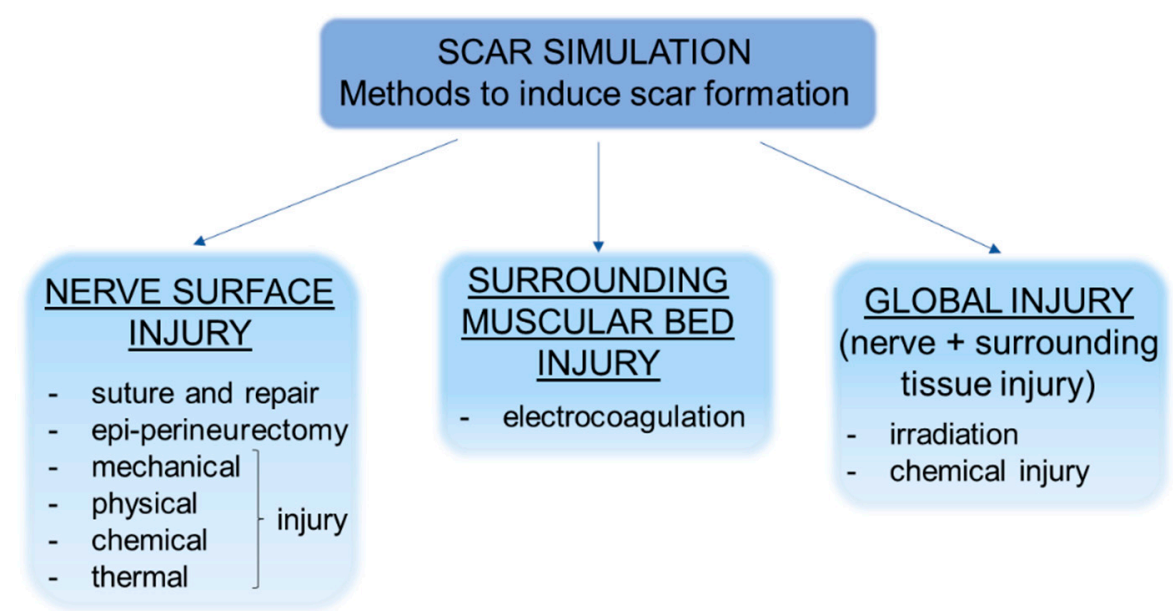

Figure 1. Representative scheme showing the different strategies to simulate scar formation in preclinical models.

Some of the earlier papers [23-25] performed a two-stage procedure (first stage injury, second stage neurolysis and antiadhesion application), which is a more traumatic experience for animals, without evidence of increased efficacy compared to a one-stage procedure. With respect to the 3Rs statement [26], a single-stage experiment can have the same efficacy as a two-stage one. 


\section{Scar Evaluation: Methods to Evaluate Scar Formation in Experimental Models}

Different procedures can be used to evaluate scar formation, including gross examination of the scar tissue, microscopical analysis of the nerve and surrounding tissue, functional tests, and electrophysiological and biomechanical evaluations (Figure 2). All of these evaluation methods are combined differently by authors. It must also be noted that the time points analyzed are very different among the studies, ranging from few days to several months from the induction of scar formation; the research also differs according to the employed animal model.

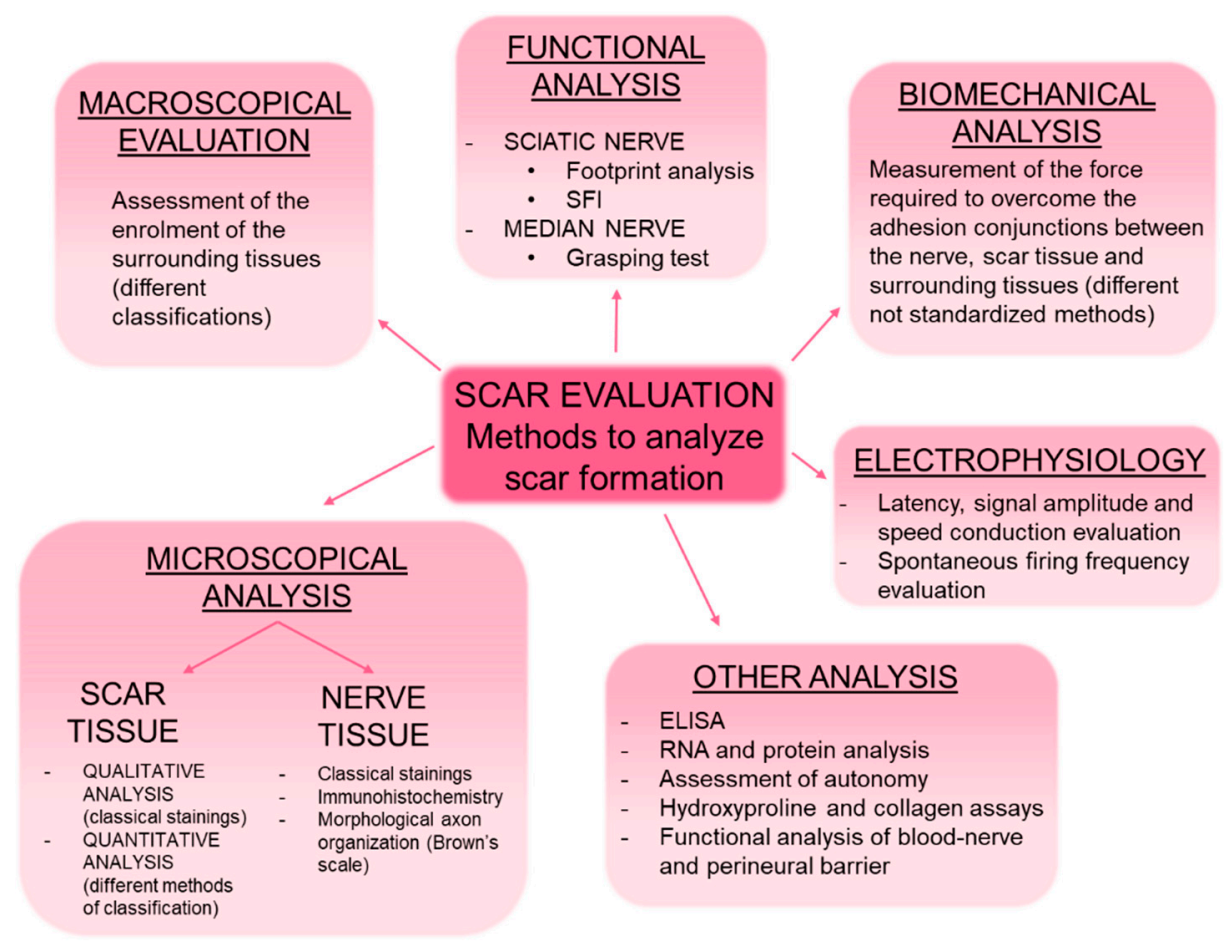

Figure 2. Representative scheme showing the different methods of evaluating scar formation in preclinical models. SFI (sciatic functional index), ELISA (enzyme-linked immunosorbent assay).

\subsection{Macroscopical Analysis}

Gross evaluation is the first fundamental step to macroscopically grade scar tissue; it aims to assess the enrolment of the surrounding tissues (including skin, muscles, and deep tissues) in the compression of the nerve and the collaboration with the newborn perineural scar tissue.

Different classifications have been proposed by different authors to evaluate the degree of scar formation. The most used and complete classification is the numeric grade scheme defined by Petersen [27]. This classification allows us to evaluate closure of skin and muscle fascia (Grade 1: skin or muscle fascia entirely closed; Grade 2: skin or muscle fascia partially open; Grade 3: skin or muscle fascia completely open) and to evaluate nerve adherence (Grade 1: no dissection or mild blunt dissection; Grade 2: some vigorous blunt dissection required; Grade 3: sharp dissection required). Another adopted grading scheme is the 4-point qualitative scale that evaluates the perineural adhesions and type of dissection required to achieve complete neurolysis, as follows: absent or thin adhesions-delicate blunt (score 0); mild adhesion-vigorous blunt (score 1); moderate adhesion-delicate sharp (score 2); severe adhesion-difficult sharp (score 3) [13,14,23,28].

Abe [17] proposed a classification of nerve adhesion based on Fontana's band (an optical manifestation of axonal undulations characteristic of peripheral nerves). They classify nerve adhesion as Group I (nonadhesion group) when the bands appear and Group II if they are not visible. Additionally, Group II is divided into Group IIa when a thickening 
of the epineurium and perineurium is observed (but not endoneurial fibrosis) and Group IIb when endoneurial fibrosis is observed.

Finally, some authors have reported the presence or absence and qualitative observations of scar tissue around the nerve without grading it.

\subsection{Microscopical Analysis: Histological Staining and Immunohistochemistry}

Microscopical analysis is employed by most authors and consists mainly of the use of different histological stainings to visualize and describe the different structures involved (not only the scar tissue but also the nerve and surrounding tissues, such as muscle), both qualitatively and quantitatively (see Tables 1-6).

\subsubsection{Analysis of the Scar Tissue}

The most employed method to highlight scar tissue is Masson's trichrome staining because it specifically marks collagen in green/blue and can be easily distinguished from other structures such as muscle fibers (stained in red), cytoplasm (light red or pink), and cell nuclei (dark brown to black).

Haematoxylin and eosin (H\&E) is the most widely used staining for histological purposes because it provides a general overview of the tissue, and it is mainly used to distinguish nerves from surrounding tissues. It is the combination of two histological stains: hematoxylin, which stains cell nuclei in blue/dark-purple, and eosin, which stains cytoplasm in pink, and other structures, including extracellular matrix and collagen, in shades of pink.

Picrosirius red is often used since it selectively highlights collagen fibers [29]; indeed, this dye allows us to visualize collagen fibers in red (specific for collagen types I and III), while the other structures are stained in yellow (nuclei, cytoplasm, muscular fibers, red blood cells). The Gordon and Sweet technique, used to reveal reticulinic acid, a collagenous tissue marker, has also been adopted, as well as chromotrop-aniline-blue, which stains collagen in blue and muscle fibers in red.

Besides a qualitative analysis of scar tissue formation, histological staining allows us to also perform quantitative analyses. One of the most used parameters to measure scar tissue is the scar tissue formation index, calculated by dividing the mean thickness of the scar tissue by the mean thickness of the nerve tissue $[18,27,28,30-41]$. Sakurai's classification of neural fibrosis [42] allows us to describe the extension of the perineural scar from epineurium to endoneurium (Grade 1: the nerve is normal; Grade 2: extraneural type; Grade 3: intraneural, epineural type; Grade 4: intraneural, perineural type; Grade 5: intraneural, endoneurial type; Grade 6: dispersive type) [17].

The number of fibroblasts/fibrocytes is another parameter that is often used, and it allows us to classify the specimens into Grade 1-less than 100 fibroblasts; Grade 2-100-150 fibroblasts; Grade 3-more than 150 fibroblasts [30,32,34,41,43,44].

The classification of Ornelas [45] has also been adopted [46,47], and it allows us to classify extraneural and intraneural fibrosis; extraneural fibrosis is classified into Grade 1-absent or minimal fibrosis; Grade 2-moderate fibrosis; Grade 3-major fibrosis. Intraneural fibrosis is classified into Grade 1 -the presence of fibrous tissue between the nerve fibers; Grade 2-fibrous tissue partially blocking the passage of nerve fibers; Grade 3-fibrous tissue completely interrupting the passage of nerve fibers.

Dam-Hieu [28] calculated the thickness of the dense scar surrounding the nerve. The largest thickness of the scar ring (ST) was measured. This value was then normalized by dividing it by the nerve diameter (ND). The authors call this value the fibrotic index (fibrotic index $=2 \mathrm{ST} / \mathrm{ND}$ ).

Other quantitative or semiquantitative analyses have also been proposed, such as the average thickness of collagen in the epineurium [14,48], the count of fibroblasts and inflammatory cells $[36,38,46]$, the thickness of the epifascicular epineurium, the amount of connective tissue in the interfascicular and epifascicular epineurium [49], and the percentage of area of staining (PAS) calculation by outlining the intraneural tissue [50-53]. 


\subsubsection{Analysis of the Nerve Tissue}

To investigate the nerve tissue specifically, Luxol fast blue, P-phenylenediamine, silver staining, the Weil method, and toluidine blue were used to describe axon distribution and highlight the myelin sheath. In particular, toluidine blue staining offers the possibility of performing morphoquantitative analyses to estimate the number of myelinated fibers, axon density, fiber and axon diameter, myelin thickness, and g-ratios (axon-diameter/fiberdiameter), which can be correlated with functional recovery [5,54].

Moreover, the longitudinal histomorphological organization of the axon at the nerve repair site can be evaluated according to the scale developed by Brown et al. [55] and adopted by several authors [31,35,36,38,56]: Grade 1 -failure, no continuity of the axons from the proximal to the distal ends; Grade 2-poor organization (interlacing or whirling appearance of the nerve fibers); Grade 3-fair organization (focal whirling appearance, focal parallel alignment); Grade 4-good organization, approaching normal (mostly parallel, without a whirling or wavy appearance); Grade 5-excellent organization of the repair site, indistinguishable from the norm.

Finally, some authors have added immunohistochemistry to classical staining methods. This method is efficient in describing nerve regeneration quality with antibodies, which specifically mark Schwann cells (anti-S100, to mark the myelin sheath) [21,36], nerve cones (anti-GAP 43) [37], or antineurofilament [21,57]. Immunohistochemistry has been also adopted to study perineural scars by using antibodies against TGF- $\beta$ markers of macrophages [58], CD68 for activated macrophages [21,37,57,59], anti-CCR7 for proinflammatory M1 macrophages [57], CD3, CD8 [21], and collagen I [60]. Additionally, antibodies against decorin, aggrecan, laminina 2, collagen IV, and fibronectin have been used [61]. Finally, Murakami [62] performed immunohistochemistry analysis on dorsal root ganglia using the inflammation marker CGRP and tissue stress ATF3 antibodies.

\subsection{Functional Analysis}

Some studies performed functional analyses, even though these analyses are not precise for scar quantification and we are not sure that they can be directly correlated to the amount of scarring observed around the nerve.

Most of the studies dealing with peripheral scarring use the sciatic nerve model, foot print analysis, and the Sciatic Function Index (SFI) as the most adopted tests [63]. Other parameters evaluated are allodynia by means of von Frey filaments [62] and walking patterns induced by pain with the CatWalk system [21,51,62].

The only study that used the median nerve model tested the function of the nerve by means of the grasping test [16].

\subsection{Electrophysiological Study}

Electrophysiology is another test that can be used in order to analyze the formation of scars around the nerve. Indeed, compression around a nerve causes pathophysiological changes that can be registered with this assessment.

The electrophysiological analysis is based on compound motor action potential (CMAP). Different aspects of electrical activity can be registered, such as latency, signal amplitude, and speed conduction. These parameters correlate with nerve conduction; the more the scar is present, the more these parameters are altered.

Another assessment that is useful to evaluate is the frequency of spontaneous firing because it has been demonstrated to be related to nerve suffering: the more frequent the firing is, the more the nerve is suffering [64].

Zuijdendorp [22] and its colleagues performed the evaluation by means of magnetoneurography of first peak amplitude, peak-peak amplitude, area, and conduction velocity over the nerve segment between the stimulation and the recording site.

Recently, the combination of electrodiagnostic evaluation, with the commonly used grasping test (reflex-based gross motor function) and the staircase test (skilled forelimb reaching), has been found to produce results with high translatability [65]. 


\subsection{Biomechanical Analysis}

Biomechanical analysis gives an objective evaluation of scar tissue formation and consists of measuring the force required to overcome the adhesion conjunctions between the nerve, scar tissue, and surrounding tissues. Different methods have been proposed, but the results obtained represent, with variability according to the precision of the utilized instruments, a quantitative expression of newborn scar tissue.

Dumanian [66] and his colleagues were the first to describe a method and device to measure the strength of nerve adhesion to surrounding muscles. They used a standard alligator clamp placed on the nerve and a force transducer connected, in turn, to a micrometer. The micrometer was distracted in $1 \mathrm{~mm}$ increments. The measurement is continued until final failure of the nerve or nerve pullout from the clamp.

Another method is to mount the nerve proximal stump on a digital force gauge using a suture connected to the load cell; then, the nerve is subjected to traction at a rate of 2 $\mathrm{cm} / \mathrm{min}$ (or $1 \mathrm{~cm} / \mathrm{s}$ ) [24] until its complete detachment from the neural bed; the ultimate strength is recorded [57,67-69].

A different way consists of transecting both the proximal and distal ends of the nerve; the proximal end is then interconnected to a force transducer, which is connected, in turn, to a motorized drive with a constant extension rate of $29 \mathrm{~mm} / \mathrm{min}$. The force required to pull the nerve segment out of its tissue bed is recorded [10,22].

In another paper, after nerve and surrounding tissue removal from the animal, the distal end of the nerve was held by a clamp to the cross-head of an Instron machine. The subsequent cross-head movement (at a rate of $10 \mathrm{~mm} / \mathrm{min}$ ) then gradually peeled the nerve away from the adhesion site, and the maximum peeling force was recorded [15].

Finally, some recent papers have described a simple and cheap method that consists of connecting the nerve to a plastic can that is gradually filled with water at a constant flow of $100 \mathrm{~mL} / \mathrm{min}$. The adhesion force is obtained from the grams of water at the break moment $[20,70,71]$.

\subsection{Other Analysis}

Other types of analyses have also been described, such as enzyme-linked immunosorbent assays (ELISAs) to evaluate neurothophic factor concentration [62], RNA and protein analysis [61], assessment of autotomy [37], functional analysis of the blood-nerve barrier and the perineurial barrier [69], and hydroxyproline and collagen assays [60].

In vitro culture of rat skin fibroblasts to test the efficacy of drug administration has also been described [58].

Histological staining can also be used to describe muscle tissue organization in order to evaluate atrophy and fibrous degeneration of the innervated muscles $[13,49,57]$. Moreover, atrophy is often investigated by measuring muscle wet weight. Finally, transmission electron microscopy has been adopted to describe the ultrastructure of nerve tissue and surrounding tissues $[30,39,48,60,72]$.

\section{How to Prevent Scar Formation? An Overview on Different Antiadhesion Devices}

Every surgical practice on peripheral nerves is followed by postsurgical scar tissue formation. In order to limit this event, surgeons apply different procedures such as local or free tissue transfer and antiadherent items of different origins. There are many different kinds of antiadhesion devices, composed of different materials with different ways of application, but there is no evidence of their efficacies. Below is an overview of the different antiadhesion devices tested so far in experimental models.

\subsection{Polysaccharide-Based Devices}

Different polysaccharides were used as antiscarring agents, and the available preclinical studies on the polysaccharides-based devices are reported in Table 2. 
Table 2. Polysaccharide-based antiscarring agents.

\begin{tabular}{|c|c|c|c|c|c|}
\hline Reference & $\begin{array}{l}\text { Method to Induce Scar } \\
\text { Formation }\end{array}$ & Agent & $\begin{array}{c}\text { Animal and Nerve } \\
\text { Model }\end{array}$ & Analyses & Results \\
\hline Hachinota et al., 2020 [73] & $\begin{array}{l}\text { Section of transvers } \\
\text { carpal ligament, } \\
\text { excision of median } \\
\text { nerve bed, suture of } \\
\text { carpal ligament }\end{array}$ & $\begin{array}{l}\text { Alginic acid-based gel } \\
\text { formulation }\end{array}$ & $\begin{array}{l}\text { Japanese White Rabbit } \\
\text { Median nerve }\end{array}$ & $\begin{array}{l}\text { - } \quad \text { Electrophysiological evaluation }(1,2, \\
\text { 3, } 6 \text { weeks) } \\
\text { - Macroscopic evaluation (adhesion } \\
\text { scoring system modified from } \\
\text { Palatinsky ones) } \\
\text { - Histology on tissues excised after } \\
\text { electrophysiological evaluation } \\
\text { (H\&E staining) }\end{array}$ & $\begin{array}{l}\text { Longer latency, not significant, in the } \\
\text { control group. Lower adhesion score } \\
\text { values in the treatment group at 2-3-6 } \\
\text { weeks, more scar tissue in the control } \\
\text { group. More severe perineural } \\
\text { fibrosis in the control group. }\end{array}$ \\
\hline
\end{tabular}

- $\quad$ Functional evaluation (SFI) $(4,8$, 12 weeks)

- $\quad$ Gross Evaluation (Petersen's classification) $(4,8,12$ weeks $)$

Li et al., 2018 [48]

Crush injury

Chitosan conduit (CC); hyaluronic acid (HA); $\mathrm{CC}+\mathrm{HA}$

Sprague-Dawley RatSciatic nerve
- Electrophysiological evaluation (12 weeks)

- Histology on nerve and surrounding tissue (H\&E, Masson's trichrome and Toluidine Blue) $(4,8,12$ weeks $)$

- $\quad$ Ultrastructural evaluation (TEM) (4, 8,12 weeks)
Both chitosan and HA inhibited extraneural scarring, promoted nerve regeneration, increased nerve conduction velocity, and improved the recovery of nerve function.
- Gross evaluation (Petersen's classification) (12 weeks)

- $\quad$ Muscle wet weight (12 weeks)

Mekaj et al., 2017 [36] Section + suture
Male European Rabbit - $\quad$ Histology on nerve and surrounding

Sciatic nerve tissue (H\&E, Masson's trichrome) (12 weeks)

- $\quad$ Nerve IHC (S100) (12 weeks)
Reduction scar around the nerve, both macroscopically and microscopically. Increased nerve diameter. Higher gastrocnemius mass. Improved microstructural organization. Higher expression of $\mathrm{S} 100$. 
Table 2. Cont.

\begin{tabular}{|c|c|c|c|c|c|}
\hline Reference & $\begin{array}{l}\text { Method to Induce Scar } \\
\text { Formation }\end{array}$ & Agent & $\begin{array}{c}\text { Animal and Nerve } \\
\text { Model }\end{array}$ & Analyses & Results \\
\hline Tos et al., 2016 [71] & Burning & $\begin{array}{l}\text { Carboxymethylcellulose } \\
\text { (CMC)-polyethylene } \\
\text { oxide (PEO) gel }\end{array}$ & $\begin{array}{l}\text { CD1 Mouse } \\
\text { Sciatic nerve }\end{array}$ & $\begin{array}{ll}- & \text { Biomechanical analysis (3 weeks) } \\
- & \text { Histology on nerve and surrounding } \\
\text { tissue (Picrosirius staining) (3 weeks) }\end{array}$ & $\begin{array}{l}\text { Reduction in scar tissue after } \\
\text { CMC-PEO gel application. The } \\
\text { qualitative histological analysis } \\
\text { supported the biomechanical findings } \\
\text { depicting the pattern of scar tissue. }\end{array}$ \\
\hline Urano et al., 2016 [68] & $\begin{array}{l}\text { Enwrapping with } \\
\text { silicon tube (nerve } \\
\text { compression) }\end{array}$ & $\begin{array}{l}\text { CMC-phosphatidyl- } \\
\text { ethanolamine } \\
\text { (PE) }\end{array}$ & $\begin{array}{l}\text { Male Lewis RatSciatic } \\
\text { nerve }\end{array}$ & $\begin{array}{ll}\text { - } & \text { Electrophysiological evaluation }(1,2, \\
& 3 \text { months) } \\
\text { - } & \text { Biomechanical analysis }(1,2, \\
& 3 \text { months) } \\
- & \text { Muscle wet weight }(1,2,3 \text { months }) \\
- & \text { Histology on nerve and } \\
\text { morphometric analysis (Toluidine } \\
\text { Blue) }(1,2,3 \text { months) }\end{array}$ & $\begin{array}{l}\text { Electrophysiology showed } \\
\text { significantly quicker recovery; mean } \\
\text { wet muscle weight was constantly } \\
\text { higher; the axon area at one month } \\
\text { was twice as large as control. }\end{array}$ \\
\hline Marcol et al., 2011 [37] & Section + suture & Chitosan & $\begin{array}{l}\text { Male Wistar Rat } \\
\text { Sciatic nerve }\end{array}$ & $\begin{array}{ll}\text { - } & \text { Autotomy assessment (daily, until } \\
\text { 20th week) } \\
\text { - } \quad \text { Histology on nerve and surrounding } \\
\text { tissue (H\&E, Masson's trichrome and } \\
\text { Toluidine Blue) (20 weeks) } \\
\text { - } \quad \text { Nerve IHC (CD-68, GAP43) } \\
\text { (20 weeks) }\end{array}$ & $\begin{array}{l}\text { High incidence of amputations (about } \\
100 \% \text {, no sig. diff.). Reduction in } \\
\text { microscopical analysis of neuroma in } \\
\text { chitosan; significant reduction of scar } \\
\text { around nerve; increased mast cells } \\
\text { and macrophages in } \\
\text { chitosan.Application of the } \\
\text { microcrystalic chitosan gel is easy and } \\
\text { requires no special equipment but } \\
\text { does not influence the features of } \\
\text { neuropathic pain. }\end{array}$ \\
\hline Park et al., 2011 [38] & Section + suture & HA-CMC & $\begin{array}{l}\text { Sprague-Dawley Rat } \\
\text { Sciatic nerve }\end{array}$ & $\begin{array}{l}\text { Gross evaluation (Petersen's } \\
\text { classification) }(3,6,9,12 \text { weeks }) \\
\text { - Histology on nerve and surrounding } \\
\text { tissue (H\&E, Masson's trichrome) (3, } \\
6,9,12 \text { weeks) }\end{array}$ & $\begin{array}{l}\text { Macroscopical scar reduction. } \\
\text { Reduction of inflammation cells and } \\
\text { fibroblasts. Reduction of scar } \\
\text { formation index. Better axonal } \\
\text { organization. }\end{array}$ \\
\hline
\end{tabular}


Table 2. Cont.

\begin{tabular}{|c|c|c|c|c|c|}
\hline Reference & $\begin{array}{l}\text { Method to Induce Scar } \\
\text { Formation }\end{array}$ & Agent & $\begin{array}{c}\text { Animal and Nerve } \\
\text { Model }\end{array}$ & Analyses & Results \\
\hline $\begin{array}{l}\text { Hernández-Cortés et al., } \\
2010 \text { [74] }\end{array}$ & $\begin{array}{l}\text { Tissue aggression } \\
\text { (cauterization of muscle } \\
\text { bed) }\end{array}$ & $\begin{array}{l}\text { Oxidized regenerated } \\
\text { cellulose }\end{array}$ & $\begin{array}{l}\text { Male Sprague-Dawley } \\
\text { RatSciatic nerve }\end{array}$ & $\begin{array}{l}\text { Histology on nerve and surrounding } \\
\text { tissue (H\&E, Masson's trichrome, } \\
\text { PAS, or Syrius red) ( } 3 \text { and } 6 \text { weeks) }\end{array}$ & $\begin{array}{l}\text { No statistical differences in intra- and } \\
\text { perineural scars, which demonstrate } \\
\text { no antifibrogenic effect of oxidized } \\
\text { regenerated cellulose. Inflammatory } \\
\text { phenomena and foreign body } \\
\text { granulomatous reactions were more } \\
\text { frequently detected in oxidized } \\
\text { regenerated cellulose-treated samples. }\end{array}$ \\
\hline Yamamoto et al., 2010 [67] & $\begin{array}{l}\text { Burning muscle }+ \text { epi- } \\
\text { and perineurium } \\
\text { removal }\end{array}$ & CMC-PE & $\begin{array}{l}\text { Lewis Rat } \\
\text { Sciatic nerve }\end{array}$ & $\begin{array}{ll}- & \text { Gross evaluation (evaluation of } \\
\text { healing of skin and fascia-Grade } \\
1-3) \text { ( } 6 \text { weeks) } \\
\text { - } \quad \text { Biomechanical analysis (6 weeks) } \\
\text { - } \quad \text { Histology on nerve and surrounding } \\
\text { tissue (H\&E Masson's trichrome) }(1, \\
2,3,4,5,6 \text { weeks) } \\
\text { Electrophysiology }(2,7,20 \text {, and } \\
42 \text { days) } \\
\text { - Muscle wet weight }(2,7,20, \text { and } \\
42 \text { days) }\end{array}$ & $\begin{array}{l}\text { CMC-PE hydrogel offered superior } \\
\text { efficacy to } 1 \% \text { HA and caused no } \\
\text { delay in wound healing. Reduction of } \\
\text { macroscopical scar, reduction of scar } \\
\text { in biomechanical testing. } \\
\text { Electrophysiological and muscle } \\
\text { weight analyses demonstrated the } \\
\text { effectiveness of CMC-PE treatment } \\
\text { after extensive internal neurolysis. }\end{array}$ \\
\hline
\end{tabular}

- $\quad$ Functional evaluation (SFI) (biweekly until day 32)

- Histology on nerve and surrounding tissue and morphometric analysis (Toluidine Blue) (18, 32, 42 days)

- Ultrastructural evaluation (TEM) (18, 32, 42 days)
Qualitatively less perineural scar tissue was observed using Seprafilm. No functional or histological deleterious effects were detected with

Sciatic nerve

Biomechanical analysis (6 weeks) Magnetoneurography (5 weeks) Regenerating agents (sulfated

Female Wistar glycosaminoglycan)
RatSciatic nerve
- $\quad$ Footprint analysis $(1,7,14,17,21,24$ 28,35 and 42 days)
Seprafilm placed on intact nerves or cut and repaired nerves.

Reduction of biomechanical resistance. No differences in magnetoneurography and functional analysis were detected. 
Table 2. Cont.

\begin{tabular}{|c|c|c|c|c|c|}
\hline Reference & $\begin{array}{c}\text { Method to Induce Scar } \\
\text { Formation }\end{array}$ & Agent & $\begin{array}{c}\text { Animal and Nerve } \\
\text { Model }\end{array}$ & Analyses & Results \\
\hline Dam-Hieu et al., 2005 [28] & $\begin{array}{l}\text { Abrasive injury/section } \\
+ \text { suture }\end{array}$ & $\begin{array}{l}\text { Auto cross-linked } \\
\text { polysaccharide (ACP) } \\
\text { with different viscosity }\end{array}$ & $\begin{array}{l}\text { Male Sprague-Dawley } \\
\text { Rat } \\
\text { Sciatic nerve }\end{array}$ & $\begin{array}{l}\text { - Gross evaluation (4-point qualitative } \\
\text { Scale) (4 weeks) } \\
\text { - Histology on nerve and surrounding } \\
\text { tissue (H\&E, Masson's trichrome, } \\
\text { Gordon and Sweets stain and } \\
\text { Toluidine Blue) (4 weeks) }\end{array}$ & $\begin{array}{l}\text { Significant reduction of scar tissue } \\
\text { formation was observed through } \\
\text { macro and micro analyses. }\end{array}$ \\
\hline Ohsumi et al., 2005 [69] & Burning & Alginate sol & $\begin{array}{l}\text { Lewis Rat } \\
\text { Sciatic nerve }\end{array}$ & $\begin{array}{l}\text { - Histology on nerve and surrounding } \\
\text { tissue (H\&E Masson's trichrome) (1, } \\
\text { 2,3,4,5,6 weeks) } \\
\text { - } \quad \text { Functional analysis of the } \\
\text { blood-nerve barrier and the } \\
\text { perineurial barrier ( } 6 \text { weeks) } \\
\text { - } \quad \text { Biomechanical analysis ( } 6 \text { weeks) }\end{array}$ & $\begin{array}{l}\text { Strong inhibition of perineurial } \\
\text { granulation, recovering of the } \\
\text { perineurial barrier function, } \\
\text { antiadhesive effect. }\end{array}$ \\
\hline Ikeda et al., 2003 [24] & $\begin{array}{l}\text { Burning muscular bed }+ \\
\text { suture }\end{array}$ & HA (after 6 weeks) & $\begin{array}{l}\text { White Japanese } \\
\text { RabbitSciatic nerve }\end{array}$ & $\begin{array}{ll}\text { - } & \text { Electrophysiological evaluation } \\
\text { (6 weeks) } \\
\text { - } \quad \text { Histology on nerve and surrounding } \\
\text { tissue (Masson's trichrome) } \\
\text { (6 weeks) } \\
\text { - } \quad \text { Biomechanical analysis (6 weeks) }\end{array}$ & $\begin{array}{l}\text { Significant latency reduction. } \\
\text { Qualitative reduction of scar in } \\
\text { microscopical analysis. No significant } \\
\text { reduction in biomechanical analysis. }\end{array}$ \\
\hline
\end{tabular}


Table 2. Cont.

\begin{tabular}{|c|c|c|c|c|c|}
\hline Reference & $\begin{array}{c}\text { Method to Induce Scar } \\
\text { Formation }\end{array}$ & Agent & $\begin{array}{c}\text { Animal and Nerve } \\
\text { Model }\end{array}$ & Analyses & Results \\
\hline Ozgenel et al., 2003 [35] & Section + suture & HA & $\begin{array}{l}\text { Male Sprague-Dawley } \\
\text { Rat } \\
\text { Sciatic nerve }\end{array}$ & $\begin{array}{ll}\text { - } & \text { Functional evaluation (SFI) (every } \\
\text { two weeks until week 12) } \\
\text { - } \quad \text { Gross evaluation (Petersen's } \\
\text { classification) }(4,12 \text { weeks) } \\
\text { - } \quad \text { Electrophysiological evaluation } \\
\text { (12 weeks) } \\
\text { - } \quad \text { Wet muscle weight (12 weeks) } \\
\text { - } \quad \text { Histology on nerve and surrounding } \\
\text { tissue (Masson's trichrome } 4,12 \\
\text { weeks, H\&E, Weil method for } \\
\text { morphometric analysis) (12 weeks) }\end{array}$ & $\begin{array}{l}\text { Significant reduction in scarring, } \\
\text { better conduction velocities, increased } \\
\text { axon and fiber diameter, and faster } \\
\text { functional recovery. }\end{array}$ \\
\hline Adanali et al., 2003 [49] & Section + suture & $\mathrm{HA}-\mathrm{CMC}$ & $\begin{array}{l}\text { White New Zealand } \\
\text { Rabbit } \\
\text { Sciatic nerve }\end{array}$ & $\begin{array}{ll}\text { - } & \text { Gross evaluation (qualitative) } \\
\text { - } 3 \text { months) } \\
\text { Electrophysiological evaluation } \\
\text { - } 3 \text { months) } \\
\text { Histology on muscle (H\&E, } \\
\text { Masson's trichrome) (3 months) } \\
\text { - } \quad \text { Histology on nerve and surrounding } \\
\text { tissue (H\&E, Masson's trichrome, } \\
\text { Toluidine Blue) (3 months) }\end{array}$ & $\begin{array}{l}\text { Macroscopically reduction of scar } \\
\text { tissue around the nerve. Increased } \\
\text { quality of myelin sheets and the } \\
\text { number of axons. }\end{array}$ \\
\hline
\end{tabular}

SFI (Sciatic functional index), TEM (Transmission Electron Microscope), IHC (Immunohistochemistry). 


\subsubsection{Hyaluronic acid}

Hyaluronic acid is a glycosaminoglycan that is widely found in the body of all living organisms as it is an important extracellular matrix component. Since it does not exhibit species or tissue specificity and is biodegradable in vivo, it is often used as an ideal biomaterial. It has been demonstrated that hyaluronic acid reduces epineural and extraneural scar formation $[36,38,67]$. Additionally, biomechanical reduction of scar tissue has been documented [10], together with an improvement of latency [24].

\subsubsection{Carboxymethylcellulose}

Carboxymethylcellulose is another biocompatible polysaccharide that acts as a physical barrier and can reduce scar formation in the central nervous system; it has been demonstrated that carboxymethylcellulose, in association with phosphatidylethanolamine, reduces peripheral nerve scarring and biomechanical resistance [67,71]. It has also been used in association with hyaluronic acid; additionally, in this case, it reduces scar formation, reduces inflammation cells and fibroblasts, and leads to better axonal organization [38,72], together with an increase in the quality of myelin sheets and the number of axons [49].

\subsubsection{Chitosan}

Chitosan is a polysaccharide obtained by partial deacetylation of chitin. It has wellknown advantageous properties, such as lack of toxicity and biocompatibility, biodegradability, and antimicrobial properties. Various forms of chitosan can be produced and microcrystallic chitosan gel applied to the proximal stump of a transected sciatic nerve has been shown to reduce the incidence and size of the neuroma and the formation of extraneural fibrosis [37]. It has also been used in the form of conduit in association with hyaluronic acid, and it has been demonstrated to reduce nerve scarring and promote nerve regeneration and recovery [48].

\subsubsection{Other polysaccharides}

Few papers have tested other polysaccharides, such as oxidized regenerated cellulose [74], regenerating agent OTR4120 [22], cross-linked polysaccharides [28], or alginate $[69,74]$.

Oxidized regenerated cellulose is a chemically altered form of cellulose used mainly as a hemostatic agent. It has been shown to not give an advantage to the prevention of nerve fibrosis; on the contrary, it interferes with healing by increasing inflammatory phenomena and granulomatous reactions [74]. Other polysaccharides have demonstrated their efficacy in the reduction of scarring through biomechanical and macro- and microscopical testing $[22,28,69,74]$.

\subsection{Collagen-Based Devices}

The available preclinical studies on collagen-based devices are reported in Table 3. 
Table 3. Collagen-based antiscarring agents.

\begin{tabular}{|c|c|c|c|c|c|}
\hline Reference & $\begin{array}{l}\text { Method to Induce Scar } \\
\text { Formation }\end{array}$ & Agent & $\begin{array}{c}\text { Animal and Nerve } \\
\text { Model }\end{array}$ & Analyses & Results \\
\hline Colonna et al., 2019 [16] & Section + suture & $\begin{array}{l}\text { Collagen sheath derived } \\
\text { from an acellular } \\
\text { hypoallergenic dermal } \\
\text { matrix (OrACELL) }\end{array}$ & $\begin{array}{l}\text { Female Wistar Rat } \\
\text { Median nerve }\end{array}$ & $\begin{array}{ll}\text { - } & \text { Functional evaluation (grasping test) } \\
\text { (every two months) } \\
\text { - } \quad \text { Histology on nerve and surrounding } \\
\text { tissue and morphometric analysis } \\
\text { (Toluidine Blue) (7 months) }\end{array}$ & $\begin{array}{l}\text { Axon diameter was higher in the } \\
\text { treated group. No significant } \\
\text { differences in the functional test } \\
\text { were observed. }\end{array}$ \\
\hline Lee et al., 2014 [76] & Section + suture & $\begin{array}{l}\text { Collagen-based film } \\
\text { (NeuraGen) }\end{array}$ & $\begin{array}{l}\text { Male Wistar Rat } \\
\text { Sciatic nerve }\end{array}$ & $\begin{array}{ll}\text { - } & \text { Electrophysiological evaluation } \\
\text { (12 weeks) } \\
\text { - } \quad \text { Wet muscle weight (12 weeks) } \\
\text { - } \quad \text { Histology on nerve and surrounding } \\
\text { tissue and morphometric analysis } \\
\text { (Toluidine Blue) (12 weeks) }\end{array}$ & $\begin{array}{l}\text { Reduction of scar in microscopical } \\
\text { analysis, although the } \\
\text { scar-decreasing effect of } \\
\text { bioabsorbable nerve wrap did } \\
\text { not translate into a better motor } \\
\text { nerve recovery. }\end{array}$ \\
\hline Mathieu et al., 2012 [47] & Section + suture & $\begin{array}{l}\text { Collagen membrane } \\
\text { and vein wrapping }\end{array}$ & $\begin{array}{l}\text { Female Wistar Rat } \\
\text { Sciatic nerve }\end{array}$ & $\begin{array}{l}\text { - Gross evaluation (Petersen's } \\
\text { classification) (12 weeks) } \\
\text { - Histology on nerve and surrounding } \\
\text { tissue (Masson's } \\
\text { trichrome-extraneural and } \\
\text { intraneural fibrosis, foreign } \\
\text { body reaction) }\end{array}$ & $\begin{array}{l}\text { The collagen membrane was } \\
\text { effective in reducing neural scar } \\
\text { formation. Autologous vein } \\
\text { wrapping also showed a favorable } \\
\text { effect in this indication despite less } \\
\text { successful histological outcomes. }\end{array}$ \\
\hline Kim et al., 2010 [75] & Section + suture & Collagen wrap & $\begin{array}{l}\text { Sprague-Dawley Rat } \\
\text { Sciatic nerve }\end{array}$ & $\begin{array}{ll}\text { - } & \text { Gross evaluation (Petersen's } \\
\text { classification) ( } 3 \text { months) } \\
\text { - } \quad \text { Histology on nerve and surrounding } \\
\text { tissue and morphometric analysis } \\
\text { (Toluidine Blue) ( } 3 \text { months) }\end{array}$ & $\begin{array}{l}\text { Significant reduction of inner } \\
\text { epineurium thickness in the } \\
\text { treated group. }\end{array}$ \\
\hline
\end{tabular}


Table 3. Cont.

\begin{tabular}{|c|c|c|c|c|c|}
\hline Reference & $\begin{array}{c}\text { Method to Induce Scar } \\
\text { Formation }\end{array}$ & Agent & $\begin{array}{c}\text { Animal and Nerve } \\
\text { Model }\end{array}$ & Analyses & Results \\
\hline Isla et al., 2003 [13] & $\begin{array}{l}\text { Section }+ \text { suture or } \\
\text { repair with silastic tube }\end{array}$ & $\mathrm{ADCON} / \mathrm{TN}$ & $\begin{array}{l}\text { Male Wistar Rat } \\
\text { Ulnar nerve }\end{array}$ & $\begin{array}{l}\text { - } \quad \text { Gross evaluation (4-point qualitative } \\
\text { Scale) (3 months) } \\
\text { - Histology on nerve, surrounding } \\
\text { tissue and muscle (H\&E, Masson's } \\
\text { trichrome) (3 months) }\end{array}$ & $\begin{array}{l}\text { Significant reduction of fibrosis. No } \\
\text { differences in terms of fiber density. }\end{array}$ \\
\hline Palatinsky et al., 1997 [23] & $\begin{array}{l}\text { Scratch; a second } \\
\text { neurolysis performed } 4 \\
\text { weeks later }\end{array}$ & $\begin{array}{l}\text { ADCON/TN (applied } \\
\text { after the second } \\
\text { neurolysis) }\end{array}$ & $\begin{array}{l}\text { Sprague } \\
\text { Dawley Rat } \\
\text { Sciatic nerve }\end{array}$ & $\begin{array}{l}\text { - Gross evaluation (4-point qualitative } \\
\text { Scale) (4 and } 8 \text { weeks) } \\
\text { Histology on nerve and surrounding } \\
\text { tissue (H\&E, Masson's trichrome, } \\
\text { phenylenediamine staining) (4 and } \\
8 \text { weeks) }\end{array}$ & $\begin{array}{l}\text { Significant reduction of composite } \\
\text { score (macroscopical evaluation). No } \\
\text { statistical difference in } \\
\text { axons diameter. }\end{array}$ \\
\hline Petersen et al., 1996 [27] & $\begin{array}{l}\text { External neurolysis, } \\
\text { abrasive injury on } \\
\text { muscle and nerve, } \\
\text { section + suture }\end{array}$ & $\mathrm{ADCON} / \mathrm{TN}$ & $\begin{array}{l}\text { Lewis's Albino Rat } \\
\text { Sciatic nerve }\end{array}$ & $\begin{array}{ll}\text { - } & \text { Gross Evaluation (Petersen's } \\
\text { classification) (4 and } 6 \text { weeks) } \\
\text { - } \quad \text { Electrophysiological evaluation (4 } \\
\text { and } 6 \text { weeks) } \\
\text { - } \quad \text { Histology on nerve and surrounding } \\
\text { tissue (H\&E, silver stain, van } \\
\text { Gieson's stain, Toluidine blue for } \\
\text { morphometric analysis) (4 and } \\
6 \text { weeks) }\end{array}$ & $\begin{array}{l}\text { Significant reduction of scar tissue; } \\
\text { no differences in } \\
\text { morphometrical analysis. }\end{array}$ \\
\hline
\end{tabular}


The use of ADCON-T/N, a bioabsorbable gel composed of a polyglycan ester in a phosphate-buffered saline solution, showed a significant reduction of scar formation with no residual implant material $[13,23,27]$. Additionally, the use of collagen-based film wrapped around the suture stitches showed a reduction in epineural and perineural scar tissue formation $[47,75,76]$. Finally, a recent study showed that a collagen sheath derived from an acellular hypoallergenic dermal matrix wrapped around the suture leads to better nerve regeneration in terms of axon diameter [16].

\subsection{Autologous Devices}

Different autologous devices were used as antiadhesion devices, and the results of the preclinical studies are reported in Table 4.

Table 4. Autologous tissues used as antiscarring agents.

\begin{tabular}{|c|c|c|c|c|c|c|}
\hline Reference & $\begin{array}{l}\text { Method to } \\
\text { Induce Scar } \\
\text { Formation }\end{array}$ & Agent & $\begin{array}{l}\text { Animal and } \\
\text { Nerve Model }\end{array}$ & & Analyses & Results \\
\hline $\begin{array}{l}\text { Cherubino et al., } \\
2017 \text { [70] }\end{array}$ & Burning & Fat Graft & $\begin{array}{l}\text { CD1 nude Mouse } \\
\text { Sciatic nerve }\end{array}$ & $\begin{array}{l}- \\
-\end{array}$ & $\begin{array}{l}\text { Biomechanical analysis } \\
\text { (4 weeks) } \\
\text { Histology on nerve and } \\
\text { surrounding tissue } \\
\text { (Picrosyrius red) (4 weeks) }\end{array}$ & $\begin{array}{l}\text { No significant difference } \\
\text { in biomechanical analysis. } \\
\text { Reduction of scar } \\
\text { observed through } \\
\text { microscopical analysis }\end{array}$ \\
\hline $\begin{array}{l}\text { Baltu et al., } 2017 \\
\text { [46] }\end{array}$ & Epineurectomy & $\begin{array}{l}\text { Buccal mucosa } \\
\text { graft }\end{array}$ & $\begin{array}{l}\text { Female } \\
\text { Sprague-Dawley } \\
\text { Rat } \\
\text { Sciatic nerve }\end{array}$ & - & $\begin{array}{l}\text { Gross evaluation } \\
\text { (Petersen's classification) (4, } \\
8 \text { weeks) } \\
\text { Histology on nerve and } \\
\text { surrounding tissue (H\&E, } \\
\text { Masson's } \\
\text { trichrome-extraneural } \\
\text { scar tissue and } \\
\text { inflammation) (4, } 8 \text { weeks) }\end{array}$ & $\begin{array}{l}\text { Buccal mucosa graft } \\
\text { decreases postoperative } \\
\text { adhesion and scar tissue } \\
\text { formation. Higher } \\
\text { inflammation at } 4 \text { weeks. }\end{array}$ \\
\hline
\end{tabular}

\begin{tabular}{|c|c|c|c|c|c|c|}
\hline $\begin{array}{l}\text { Murakami et al., } \\
2014 \text { [62] }\end{array}$ & $\begin{array}{l}\text { Ligature on } \\
\text { sciatic nerve }\end{array}$ & Vein Wrapping & $\begin{array}{l}\text { Male Wistar Rat } \\
\text { Sciatic nerve }\end{array}$ & $\begin{array}{l}- \\
- \\
- \\
- \\
- \\
-\end{array}$ & 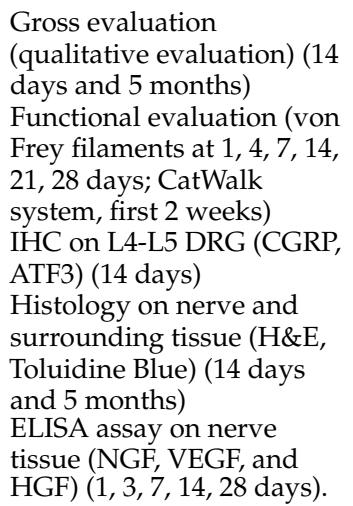 & $\begin{array}{l}\text { Significant allodynia } \\
\text { reduction. Significant } \\
\text { increase in VEGF and } \\
\text { HGF. Reduction of } \\
\text { immunoreactive cells in } \\
\text { dorsal root ganglia. }\end{array}$ \\
\hline $\begin{array}{l}\text { Meng et al., } 2011 \\
\text { [39] }\end{array}$ & Section + suture & $\begin{array}{l}\text { Amniotic } \\
\text { membrane }\end{array}$ & $\begin{array}{l}\text { Male } \\
\text { Sprague-Dawley } \\
\text { Rat } \\
\text { Sciatic nerve }\end{array}$ & $\begin{array}{l}- \\
- \\
- \\
-\end{array}$ & $\begin{array}{l}\text { Functional evaluation (SFI) } \\
\text { (weekly) } \\
\text { Gross evaluation } \\
\text { (Petersen's classification) } \\
\text { Electrophysiological tests } \\
\text { (every } 4 \text { weeks until } \\
\text { Week 12) } \\
\text { Histology on nerve and } \\
\text { surrounding tissue } \\
\text { (Picrosyrius red, toluidine } \\
\text { blue for morphometrical } \\
\text { analysis) (4,8, } 12 \text { weeks) } \\
\text { Ultrastructural evaluation } \\
\text { (TEM) (4, 8, } 12 \text { weeks) }\end{array}$ & $\begin{array}{l}\text { Significant reduction of } \\
\text { scar index. No functional } \\
\text { and morphological } \\
\text { differences were } \\
\text { observed. }\end{array}$ \\
\hline
\end{tabular}


Table 4. Cont.

\begin{tabular}{|c|c|c|c|c|c|c|}
\hline Reference & $\begin{array}{l}\text { Method to } \\
\text { Induce Scar } \\
\text { Formation }\end{array}$ & Agent & $\begin{array}{c}\text { Animal and } \\
\text { Nerve Model }\end{array}$ & & Analyses & Results \\
\hline $\begin{array}{l}\text { Kim et al., } 2010 \\
{[14]}\end{array}$ & Section + suture & $\begin{array}{l}\text { Amniotic } \\
\text { membrane }\end{array}$ & $\begin{array}{l}\text { White New } \\
\text { Zealand Rabbit } \\
\text { Ulnar nerve }\end{array}$ & - & $\begin{array}{l}\text { Gross evaluation (4-point } \\
\text { qualitative Scale) } \\
\text { ( } 3 \text { months) } \\
\text { Histology on nerve and } \\
\text { surrounding tissue } \\
\text { (Masson's trichrome-- } \\
\text { morphometrical analysis) } \\
\text { ( } 3 \text { months) }\end{array}$ & $\begin{array}{l}\text { Four-point evaluation } \\
\text { system was significant in } \\
\text { the treatment group. } \\
\text { Significant reduction of } \\
\text { scar thickness. }\end{array}$ \\
\hline $\begin{array}{l}\text { Ozgenel et al., } \\
2004 \text { [40] }\end{array}$ & Epineurectomy & $\begin{array}{l}\text { Amniotic } \\
\text { membrane + HA }\end{array}$ & $\begin{array}{l}\text { Male } \\
\text { Sprague-Dawley } \\
\text { Rat } \\
\text { Sciatic nerve }\end{array}$ & - & $\begin{array}{l}\text { Gross evaluation } \\
\text { (Petersen's classification) (4 } \\
\text { and } 12 \text { weeks) } \\
\text { Histology on nerve and } \\
\text { surrounding tissue (H\&E) } \\
\text { (4 and } 12 \text { weeks) }\end{array}$ & $\begin{array}{l}\text { Significant reduction in } \\
\text { scarring was observed } \\
\text { through microscopical } \\
\text { analysis. }\end{array}$ \\
\hline $\begin{array}{l}\text { Xu et al., } 2000 \\
{[25]}\end{array}$ & $\begin{array}{l}\text { Silastic tube } \\
\text { around the nerve }\end{array}$ & $\begin{array}{l}\text { Vein wrapping } \\
\text { (after } 8 \text { months } \\
\text { from nerve } \\
\text { compression) }\end{array}$ & $\begin{array}{l}\text { Sprague-Dawley } \\
\text { Rat } \\
\text { Sciatic nerve }\end{array}$ & $\begin{array}{l}- \\
-\end{array}$ & $\begin{array}{l}\text { Functional evaluation (SFI) } \\
(4,8,12,24 \text {, and } 48 \text { weeks) } \\
\text { Gross Evaluation } \\
\text { (qualitative evaluation) ( } 4 \text {, } \\
8,12,24 \text {, and } 48 \text { weeks) } \\
\text { Electrophysiological tests } \\
\text { (4, 8, 12, 24, and } 48 \text { weeks) } \\
\text { Histology on nerve and } \\
\text { surrounding tissue (H\&E, } \\
\text { Masson's trichrome, silver } \\
\text { staining, toluidine blue) (4, } \\
8,12,24, \text { and } 48 \text { weeks) }\end{array}$ & $\begin{array}{l}\text { Significant improvement } \\
\text { in functional analysis. } \\
\text { Electromyography and } \\
\text { microscopical analysis } \\
\text { showed no significant } \\
\text { scar reduction. }\end{array}$ \\
\hline $\begin{array}{l}\text { Dumanian et al., } \\
1999 \text { [66] }\end{array}$ & Epineurectomy & Fat graft & $\begin{array}{l}\text { Sprague-Dawley } \\
\text { Rat } \\
\text { Sciatic nerve }\end{array}$ & - & $\begin{array}{l}\text { Biomechanical analysis } \\
\text { ( } 2 \text { months) } \\
\text { Histology on nerve and } \\
\text { surrounding tissue } \\
\text { (Masson's trichrome) } \\
\text { (2 months) }\end{array}$ & $\begin{array}{l}\text { Significant reduction of } \\
\text { nerve stiffness in } \\
\text { biomechanical analysis. } \\
\text { Insignificant reduction of } \\
\text { scar thickness in } \\
\text { microscopical analysis. } \\
\text { Higher but not significant } \\
\text { incidence of neuropathy } \\
\text { in fat-graft group. }\end{array}$ \\
\hline
\end{tabular}

SFI (Sciatic functional index), TEM (Transmission Electron Microscope), IHC (Immunohistochemistry), H\&E (Hematoxylin Eosin staining), CGRP (Calcitonin gene related peptide), ATF3 (Activating transcription factor 3), VEGF (vascular endothelial growth factor), HGF (Hepatocyte growth factor), NGF (Nerve growth factor).

\subsubsection{Amniotic Membrane}

The amniotic membrane is the inner layer of fetal membranes; it is composed of an inner layer of epithelial cells on a thick basement membrane. It is nonimmunogenic, and it has been demonstrated to reduce inflammation, inhibit vascularization, combat infection, and reduce scarring. It is widely used in multiple fields of surgery and medicine, including skin substitute, wound care, urethral reconstruction, and repair of corneal and other tissues [77]. Its use in reducing peripheral nerve scarring has been demonstrated in different papers $[14,39,40]$.

\subsubsection{Fat Grafting}

In the last decade, adipose tissue has been widely studied in the field of regenerative medicine due to the presence of adipose-tissue-derived mesenchymal stem cells (which can differentiate into different cellular lineages) and its endocrine activity (release of adipocytokines, cytokines, transcriptional and growth factors). It is easy to access and harvest with painless procedures. The use of fat grafting in the prevention of peripheral scar tissue formation has had different results: it produces nerve stiffness reduction in biomechanical testing [66], but no significant differences were reported when compared to 
other antiadhesion devices. Moreover, in microscopical analysis, it appears to be able to reduce scar thickness [70].

\subsubsection{Vein Wrapping and Buccal Mucosa Graft}

Another autologous tissue that has been tested for scar formation prevention is vein tissue, which is harvested from the same animal (femoral vein) and wrapped in a spiral pattern around the nerve [25] or harvested from the abdominal portion of the donor animal vena cava and wrapped around the ligated nerve [62]. In both cases, it has been demonstrated to reduce scar formation and improve nerve function recovery.

Finally, the use of a buccal mucosa graft has also been proposed as an antiadherent device since it is composed of nonkeratinized epithelium with underlying connective tissue and includes type I and III collagen. It has been shown that when wrapped around the nerve, it decreases adhesion and scar tissue formation but leads to higher inflammation in the early postoperative period [46].

\subsection{Drugs}

Several drugs have been tested; the preclinical results are reported in Table 5. Most of them (aprotinin, tacrolimus, mannose-6-phosphate, doxorubicin, mitomycin C, citicoline, cytidine59-diphosphocholine-choline) were locally placed around the nerve [30-33,36,41,51,56]; others were intraepineurially injected (chondroitinase ABC) [61], intraperitoneally injected (citicoline and verapamil) [60,78], or intragastrically injected (tacrolimus) [58]. It has been shown that tacrolimus, an immunosuppressive drug used mainly after allogenic organ transplant, promotes nerve regeneration $[79,80]$ and scar tissue reduction [36,58]. Moreover, the application of other drugs is correlated with scar formation reduction either in macro- or microscopical analysis. In addition, the improvement of axon quality has been reported in some papers, together with enhanced functional results.

Table 5. Drugs used as antiscarring agents.

\begin{tabular}{|c|c|c|c|c|c|}
\hline Reference & $\begin{array}{l}\text { Method to } \\
\text { Induce Scar } \\
\text { Formation }\end{array}$ & Agent & $\begin{array}{l}\text { Animal and } \\
\text { Nerve model }\end{array}$ & Analyses & Results \\
\hline $\begin{array}{l}\text { Mekaj et al., } 2017 \\
\text { [36] }\end{array}$ & Section + suture & $\begin{array}{l}\text { Tacrolimus } \\
\text { (FK506) }\end{array}$ & $\begin{array}{l}\text { Male European } \\
\text { Rabbit } \\
\text { Sciatic nerve }\end{array}$ & $\begin{array}{ll}\text { - } & \text { Gross evaluation } \\
\text { (Petersen's classification) } \\
\text { (12 weeks) } \\
\text { - } \quad \text { Muscle wet weight } \\
\text { (12 weeks) } \\
\text { - } \quad \text { Histology on nerve and } \\
\text { surrounding tissue (H\&E, } \\
\text { Masson's trichrome) } \\
\text { Fibroblast and } \\
\text { inflammatory cell counts } \\
\text { (12 weeks) } \\
\text { Nerve IHC (S100) } \\
\text { (12 weeks) }\end{array}$ & $\begin{array}{l}\text { Scar reduction around the } \\
\text { nerve, both } \\
\text { macroscopically and } \\
\text { microscopically. } \\
\text { Increased nerve diameter. } \\
\text { Higher gastrocnemius } \\
\text { mass. Improved } \\
\text { microstructural } \\
\text { organization. Higher } \\
\text { expression of S100. }\end{array}$ \\
\hline $\begin{array}{l}\text { Zhu et al., } 2017 \\
\text { [61] }\end{array}$ & $\begin{array}{l}\text { Silicone tube } \\
\text { around the nerve }\end{array}$ & $\begin{array}{l}\text { Decompression } \\
\text { and } \\
\text { chondroitinase } \\
\text { ABC ( } 6 \text { weeks } \\
\text { after } \\
\text { compression } \\
\text { injury) }\end{array}$ & $\begin{array}{l}\text { Male } \\
\text { Sprague-Dawley } \\
\text { Rat and Male } \\
\text { C57BL/6 Mouse } \\
\text { Sciatic nerve }\end{array}$ & $\begin{array}{ll}\text { - } & \text { Electrophysiology } \\
\text { - } & \text { } 1 \text { month) } \\
\text { RNA (neuron-glial antigen } \\
\text { 2, phosphacan, brevican, } \\
\text { versican, aggrecan, and } \\
\text { decorin) and protein } \\
\text { expression (decorin, } \\
\text { aggrecan, laminina 2, } \\
\text { collagen IV, and fibronectin) } \\
\text { (1 month) } \\
\text { Nerve IHC (decorin, } \\
\text { aggrecan, laminina 2, } \\
\text { collagen IV, and fibronectin) } \\
\text { (1 month) }\end{array}$ & $\begin{array}{l}\text { Surgical decompression } \\
\text { alone does not reverse the } \\
\text { functional changes to the } \\
\text { nerve, whereas the } \\
\text { administration of } \\
\text { chondroitinase-ABC, in } \\
\text { addition to } \\
\text { decompression, resulted } \\
\text { in functional } \\
\text { improvement. }\end{array}$ \\
\hline
\end{tabular}


Table 5. Cont.

\begin{tabular}{|c|c|c|c|c|c|}
\hline Reference & $\begin{array}{l}\text { Method to } \\
\text { Induce Scar } \\
\text { Formation }\end{array}$ & Agent & $\begin{array}{l}\text { Animal and } \\
\text { Nerve model }\end{array}$ & Analyses & Results \\
\hline $\begin{array}{l}\text { Vural et al., } 2016 \\
\text { [41] }\end{array}$ & Abrasion & $\begin{array}{l}\text { Mitomycin C/ } \\
\text { Daunorubicin }\end{array}$ & $\begin{array}{l}\text { Male Wistar Rat } \\
\text { Sciatic nerve }\end{array}$ & 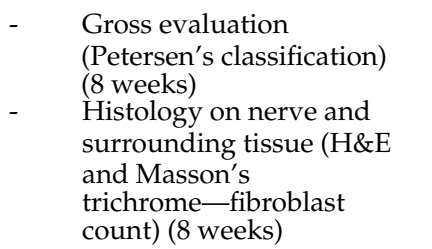 & $\begin{array}{l}\text { Macroscopically, } \\
\text { mitomycin C, and } \\
\text { daunorubicin decreased } \\
\text { adhesion. Scar tissue } \\
\text { thickness and } \\
\text { fibroblast/fibrocyte cell } \\
\text { number were reduced. }\end{array}$ \\
\hline $\begin{array}{l}\text { Xue et al., } 2016 \\
{[60]}\end{array}$ & Section + suture & $\begin{array}{l}\text { Verapamil } \\
\text { (calcium channel } \\
\text { blockers) }\end{array}$ & $\begin{array}{l}\text { Sprague-Dawley } \\
\text { Rat } \\
\text { Sciatic nerve }\end{array}$ & $\begin{array}{ll}\text { - } & \begin{array}{l}\text { Gross evaluation } \\
\text { (qualitative evaluation) (4 }\end{array} \\
\text { and } 12 \text { weeks) } \\
\text { Nerve IHC (collagen I) (4 } \\
\text { and } 12 \text { weeks) } \\
\text { - } \quad \text { Ultrastructural evaluation } \\
\text { (TEM) (4 and } 12 \text { weeks) } \\
\text { Hydroxyproline and } \\
\text { collagen assay (4 and } \\
12 \text { weeks) }\end{array}$ & $\begin{array}{l}\text { The collagen content of } \\
\text { nerve scar was } \\
\text { apparently less than that } \\
\text { of the control group; } \\
\text { more cytoplasmic vesicles } \\
\text { in the fibroblasts of the } \\
\text { treated group were } \\
\text { observed. }\end{array}$ \\
\hline $\begin{array}{l}\text { Kaplan et al., } \\
2014 \text { [78] }\end{array}$ & Section + suture & Citicoline & $\begin{array}{l}\text { Female Wistar } \\
\text { Albino Rat } \\
\text { Sciatic nerve }\end{array}$ & $\begin{array}{ll}\text { - } & \text { Functional evaluation (SFI) } \\
(4,8,12 \text { weeks) } \\
\text { - } \quad \text { Electromyography } \\
\text { (12 weeks) } \\
\text { - Gross evaluation } \\
\text { (Petersen's classification) } \\
\text { (12 weeks) } \\
\text { Histology on nerve and } \\
\text { surrounding tissue } \\
\text { (Masson's trichrome and } \\
\text { Toluidine Blue for } \\
\text { morphometrical analysis) } \\
\text { (12 weeks) }\end{array}$ & $\begin{array}{l}\text { Improvement of SFI. } \\
\text { Significant reduction in } \\
\text { scarring. Significant } \\
\text { increase in myelinated } \\
\text { axons in C } 900 \text { and } \\
\text { reduction of scar in the } \\
\text { treated group. }\end{array}$ \\
\hline $\begin{array}{l}\text { Que et al., } 2013 \\
\text { [58] }\end{array}$ & Section + suture & $\begin{array}{l}\text { Tacrolimus } \\
\text { (FK506) }\end{array}$ & $\begin{array}{l}\text { Male } \\
\text { Sprague-Dawley } \\
\text { Rat } \\
\text { Sciatic nerve }\end{array}$ & $\begin{array}{ll}\text { - } & \text { Nerve IHC (TGF- } \beta \text { ) } \\
\text { (4 weeks) } \\
\text { - } \quad \text { Histology on nerve and } \\
\text { surrounding tissue } \\
\text { (Masson's trichrome) } \\
\text { (4 weeks) } \\
-\quad \text { In vitro analysis }\end{array}$ & $\begin{array}{l}\text { FK506 has a valid effect } \\
\text { on scar formation } \\
\text { reduction in sciatic } \\
\text { nerve-injured rat by } \\
\text { inducing fibroblast } \\
\text { apoptosis. }\end{array}$ \\
\hline $\begin{array}{l}\text { Ngeow et al., } \\
2011 \text { [50] }\end{array}$ & Section + suture & $\begin{array}{l}\text { Triamcinolone } \\
\text { acetonide, } \\
\text { Interleukin-10 (IL } \\
\text { 10), mannose-6- } \\
\text { phosphate } \\
(\mathrm{M} 6 \mathrm{P})\end{array}$ & $\begin{array}{l}\text { C57 Black-6 } \\
\text { Mouse } \\
\text { Sciatic nerve }\end{array}$ & $\begin{array}{ll}\text { - } & \text { Electrophysiological } \\
\text { evaluation (6 and } 12 \text { weeks) } \\
\text { - } \quad \text { Functional evaluation } \\
\text { (CatWalk) }(1,3,6,9, \text { and } 12 \\
\text { weeks } \\
\text { - } \quad \text { Histology on nerve and } \\
\text { surrounding tissue } \\
\text { (Picrosirius staining) (6 and } \\
12 \text { weeks) }\end{array}$ & $\begin{array}{l}\text { The percentage of } \\
\text { scarring was not } \\
\text { significantly different } \\
\text { between methods in } \\
\text { microscopical analysis. } \\
\text { Reduction of compound } \\
\text { action potential in } \\
\text { triamcinolone and M6P } \\
200 \text { was observed } \\
\text { through EMG. }\end{array}$ \\
\hline $\begin{array}{l}\text { Ngeow et al., } \\
2011[51]\end{array}$ & Section + suture & $\begin{array}{l}\text { Mannose-6- } \\
\text { phosphate }\end{array}$ & $\begin{array}{l}\text { C57 Black- } 6 \text { mice } \\
\text { Sciatic nerve }\end{array}$ & $\begin{array}{ll}\text { - } & \text { Electrophysiological } \\
\text { evaluation (6 and } 12 \text { weeks) } \\
\text { - } \quad \text { Histology on nerve and } \\
\text { surrounding tissue } \\
\text { (Picrosirius staining) (6 and } \\
\text { 12 weeks) } \\
\text { Functional evaluation } \\
\text { (CatWalk) }(1,3,6,9 \text { and } \\
12 \text { weeks }\end{array}$ & $\begin{array}{l}\text { Larger compound action } \\
\text { potential and better } \\
\text { functional recovery in } \\
\text { early evaluation. } \\
\text { Reduction in collagen } \\
\text { staining. }\end{array}$ \\
\hline
\end{tabular}


Table 5. Cont.

\begin{tabular}{|c|c|c|c|c|c|c|}
\hline Reference & $\begin{array}{l}\text { Method to } \\
\text { Induce Scar } \\
\text { Formation }\end{array}$ & Agent & $\begin{array}{l}\text { Animal and } \\
\text { Nerve model }\end{array}$ & & Analyses & Results \\
\hline $\begin{array}{l}\text { Aslan et al., } 2011 \\
\text { [56] }\end{array}$ & $\begin{array}{l}\text { Section + suture } \\
\text { (immediate or } 3 \\
\text { days later) }\end{array}$ & $\begin{array}{l}\text { CDP-choline, } \\
\text { cytidine, choline, } \\
\text { or } \\
\text { cytidine-choline } \\
\text { (during nerve } \\
\text { repair) }\end{array}$ & $\begin{array}{l}\text { Female } \\
\text { Sprague-Dawley } \\
\text { Rat } \\
\text { Sciatic nerve }\end{array}$ & - & $\begin{array}{l}\text { Functional evaluation (SFI) } \\
(4,8,12 \text { weeks) } \\
\text { Gross evaluation } \\
\text { (Petersen's classification) } \\
\text { (12 weeks) } \\
\text { Histology on nerve and } \\
\text { surrounding tissue (H\&E, } \\
\text { Weil method for } \\
\text { morphometrical analysis) } \\
\text { (12 weeks) }\end{array}$ & $\begin{array}{l}\text { Treatment with } \\
\text { CDP-choline or } \\
\text { cytidine-choline reduced } \\
\text { scar formation and } \\
\text { decreased nerve } \\
\text { adherence. }\end{array}$ \\
\hline
\end{tabular}

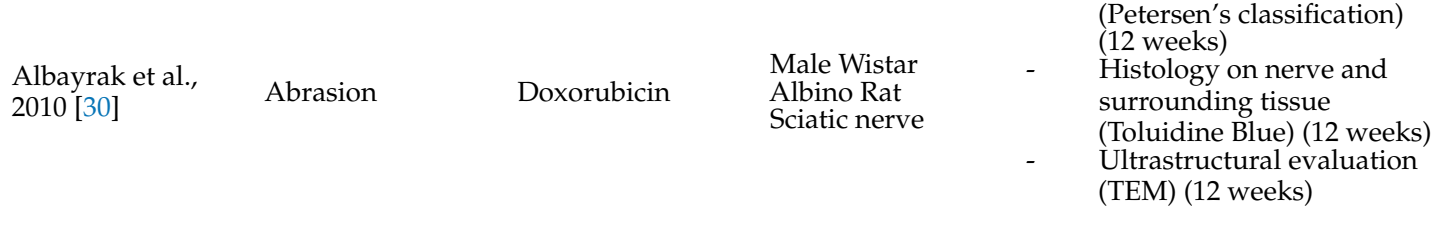

Topical application of doxorubicin effectively reduced epineural scar formation.

Functional evaluation (SF $)$
$(4,8,12$ weeks)
Gross evaluation
(Petersen's classification)
(12 weeks)
Histology on nerve and
surrounding tissue (H\&E,
Weil method for
morphometrical analysis)
(12 weeks)

Gross evaluation
(Petersen's classification)
(12 weeks)
Histology on nerve and
surrounding tissue
(Toluidine Blue) (12 week
Ultrastructural evaluation
(TEM) (12 weeks)

Electrophysiological analysis (6 weeks)

$\begin{array}{lll}\text { Atkins et al., } 2007 & \text { Section + suture } & \text { IL-10 } \\ {[53]} & & \begin{array}{l}\text { C57 Black-6 } \\ \text { MouseSciatic } \\ \text { nerve }\end{array}\end{array}$ Histology on nerve and surrounding tissue (Picrosirius staining and Toluidine Blue for morphometrical analysis) (6 weeks)

\begin{tabular}{lll}
\hline & & \\
& & \\
& & \\
Ozay et al., $2007 \quad$ Section + suture & Citicoline & $\begin{array}{l}\text { Female } \\
\text { Sprague-Dawley } \\
\text { Rat } \\
\text { Sciatic nerve }\end{array}$
\end{tabular}
Compound action potential and area of staining for collagen not significantly different compared to controls. Higher number of myelinated fibers compared to control but no difference with the other groups.

Functional evaluation (SFI)

$(4,8,12$ weeks)

Electrophysiological analysis (4,12 weeks)

Gross evaluation

(Petersen's classification)

(4 weeks)

Histology on nerve and

surrounding tissue

(Masson's trichrome, H\&E,

Weil method for

morphometrical analysis)

(4, 12 weeks)
Rats treated with

citicoline showed significantly better SFI and improvement at 12 weeks of

electromyography.

Nerves were surrounded

by only a very thin,

lucent membrane and

showed thin dark bands

of connective tissue

surrounding the nerve.

\begin{tabular}{|c|c|c|c|c|c|c|}
\hline $\begin{array}{l}\text { Ilbay et al., } 2005 \\
\text { [32] }\end{array}$ & Scratch & Mitomycin C & $\begin{array}{l}\text { Male Wistar Rat } \\
\text { Sciatic nerve }\end{array}$ & - & $\begin{array}{l}\text { Gross evaluation } \\
\text { (Petersen's classification) } \\
\text { (4 weeks) } \\
\text { Histology on nerve and } \\
\text { surrounding tissue (H\&E, } \\
\text { Masson's trichrome- } \\
\text { fibroblasts / fibrocytes } \\
\text { count) ( } 4 \text { weeks) }\end{array}$ & $\begin{array}{l}\text { Macroscopical and } \\
\text { microscopical reduction } \\
\text { of perineural adhesions } \\
\text { in the treated groups; } \\
\text { lower number of } \\
\text { fibroblast/fibrocytes. }\end{array}$ \\
\hline $\begin{array}{l}\text { Gorgulu et al., } \\
1998 \text { [33] }\end{array}$ & $\begin{array}{l}\text { External } \\
\text { neurolysis, } \\
\text { abrasive injury, } \\
\text { anastomosis }\end{array}$ & Aprotinin & $\begin{array}{l}\text { Male } \\
\text { Sprague-Dawley } \\
\text { RatSciatic nerve }\end{array}$ & $\begin{array}{l}- \\
- \\
-\end{array}$ & $\begin{array}{l}\text { Functional analysis (sciatic } \\
\text { nerve function) (weekly) } \\
\text { Gross evaluation } \\
\text { (Petersen's classification) (4, } \\
6 \text { weeks) } \\
\text { Histology on nerve and } \\
\text { surrounding tissue } \\
\text { (Masson's trichrome) (4, } \\
6 \text { weeks) }\end{array}$ & $\begin{array}{l}\text { Scar reduction after } \\
\text { aprotinin application. No } \\
\text { differences in } \\
\text { neurological tests were } \\
\text { observed. }\end{array}$ \\
\hline
\end{tabular}




\subsection{Others}

Many other devices/techniques have been investigated as antiscarring agents, and the preclinical results of these devices are reported in Table 6. A very recent study compared the efficacy of two novel biodegradable wraps made of synthetic $1 \%$ oxidized polyvinyl alcohol (OxPVA) and a leukocyte-fibrin-platelet membrane (LFPm) with the commercial product NeuraWrap, demonstrating their effectiveness in sustaining nerve regeneration, together with an absence of scar tissue/neuroma formation and significant inflammatory infiltrate [81].

Table 6. Other antiscarring agents.

\begin{tabular}{|c|c|c|c|c|c|c|}
\hline Reference & $\begin{array}{l}\text { Method to } \\
\text { induce scar } \\
\text { formation }\end{array}$ & Agent & $\begin{array}{l}\text { Animal and } \\
\text { Nerve model }\end{array}$ & & Analyses & Results \\
\hline $\begin{array}{l}\text { Kikuchi et al., } \\
2020 \text { [82] }\end{array}$ & Burning & $\begin{array}{l}\text { Polylactic acid } \\
\text { (PLA)-based } \\
\text { biodegradable } \\
\text { three-layered } \\
\text { membrane } \\
\text { (E8002) with or } \\
\text { without } \\
\text { L-ascorbic acid } \\
\text { (AA) }\end{array}$ & $\begin{array}{l}\text { Male } \\
\text { Sprague-Dawley } \\
\text { Rat } \\
\text { Sciatic nerve }\end{array}$ & - & $\begin{array}{l}\text { Motor functional (rotarod) } \\
\text { and mechanical sensitivity } \\
\text { (von Frey) evaluation } \\
\text { (before surgery and 2, 4, } \\
6 \text { weeks after). } \\
\text { Gross evaluation } \\
\text { (Petersen's classification) (6 } \\
\text { weeks) } \\
\text { Histology on nerve and } \\
\text { surrounding tissue } \\
\text { (aldehyde fuchsin } \\
\text { Masson-Goldner staining) } \\
\text { (6 weeks) }\end{array}$ & $\begin{array}{l}\text { AA in E8002 has an } \\
\text { antiadhesional effect by } \\
\text { enhancing fibrinolysis. } \\
\text { Adhesion formation was } \\
\text { lower in the group } \\
\text { containing AA. Motor } \\
\text { function and mechanical } \\
\text { sensitivity were not } \\
\text { impaired after surgery, } \\
\text { and no differences were } \\
\text { detected among groups. }\end{array}$ \\
\hline $\begin{array}{l}\text { Stocco et al., } 2019 \\
\text { [81] }\end{array}$ & Section + suture & $\begin{array}{l}\text { Wraps made of a } \\
\text { synthetic } \\
1 \% \text { oxidized } \\
\text { polyvinyl alcohol } \\
\text { (OxPVA) and a } \\
\text { leukocyte-fbrin- } \\
\text { platelet } \\
\text { membrane } \\
\text { (LFPm) } \\
\text { compared to } \\
\text { NeuroWrap }\end{array}$ & $\begin{array}{l}\text { Sprague-Dawley } \\
\text { rats } \\
\text { Sciatic nerve }\end{array}$ & - & $\begin{array}{l}\text { Functional analysis (sciatic } \\
\text { function index assessment) } \\
\text { ( } 2 \text { and } 12 \text { weeks) } \\
\text { Gross evaluation ( } 12 \text { weeks) } \\
\text { Histology on nerve and } \\
\text { surrounding tissue (H\&E, } \\
\text { IHC and Toluidine blue) } \\
\text { (12 weeks) } \\
\text { Ultrastructural analysis } \\
\text { (TEM) (12 weeks) } \\
\text { Neural collagen deposition } \\
\text { evaluation (12 weeks) }\end{array}$ & $\begin{array}{l}\text { LFPm wraps were } \\
\text { completely resorbed, } \\
\text { while residues of OxPVA } \\
\text { and NeuraWrap were } \\
\text { observed. Functional } \\
\text { recovery was achieved in } \\
\text { all groups. Additionally, } \\
\text { at the morphological } \\
\text { level, scar tissue } \\
\text { formation and } \\
\text { inflammatory infiltrate } \\
\text { were not observed. Both } \\
\text { myelinic and unmyelinic } \\
\text { axons were observed. }\end{array}$ \\
\hline $\begin{array}{l}\text { Shintani et al., } \\
2018 \text { [57] }\end{array}$ & Burning & $\begin{array}{l}\text { Polylactide } \\
\text { (PLA)-poly(e- } \\
\text { caprolactone) } \\
\text { PCL conduit and } \\
\text { HA }\end{array}$ & $\begin{array}{l}\text { Lewis Rat } \\
\text { Sciatic nerve }\end{array}$ & - & $\begin{array}{l}\text { Gross evaluation } \\
\text { (Petersen's classification) } \\
\text { (6 weeks) } \\
\text { Biomechanical examination } \\
\text { (6 weeks) } \\
\text { Electrophysiological } \\
\text { evaluation (6 weeks) } \\
\text { Muscle wet weight and } \\
\text { histology (6 weeks) } \\
\text { Nerve IHC } \\
\text { (antineurofilament, } \\
\text { anti-CD68, and anti-CCR7) } \\
\text { (6 weeks) }\end{array}$ & $\begin{array}{l}\text { Morphological properties } \\
\text { of axons were preserved } \\
\text { with PLA-PCL conduit. } \\
\text { HA was less effective for } \\
\text { nerve protection from } \\
\text { adhesion. }\end{array}$ \\
\hline $\begin{array}{l}\text { Servet et al., } 2016 \\
\text { [59] }\end{array}$ & Section + suture & $\begin{array}{l}\text { Ankaferd blood } \\
\text { stopper (ABS) } \\
\text { hemostatic agent }\end{array}$ & $\begin{array}{l}\text { Male } \\
\text { Sprague-Dawley } \\
\text { Rat } \\
\text { Sciatic nerve }\end{array}$ & - & $\begin{array}{l}\text { Electrophysiology } \\
\text { (12 weeks) } \\
\text { Histology on nerve and } \\
\text { surrounding tissue (H\&E, } \\
\text { Masson's trichrome, and } \\
\text { Luxol fast blue) (24 weeks) } \\
\text { Nerve IHC (CD68) } \\
\text { (24 weeks) }\end{array}$ & $\begin{array}{l}\text { Significant improvement } \\
\text { of latency and speed in } \\
\text { the ABS group. Other } \\
\text { results were not } \\
\text { statistically different. }\end{array}$ \\
\hline
\end{tabular}


Table 6. Cont.

\begin{tabular}{|c|c|c|c|c|c|c|}
\hline Reference & $\begin{array}{l}\text { Method to } \\
\text { induce scar } \\
\text { formation }\end{array}$ & Agent & $\begin{array}{l}\text { Animal and } \\
\text { Nerve model }\end{array}$ & & Analyses & Results \\
\hline $\begin{array}{l}\text { Okui et al., } 2010 \\
\text { [83] }\end{array}$ & $\begin{array}{l}\text { Neurolysis and } \\
\text { burning }\end{array}$ & PLA & $\begin{array}{l}\text { Male Lewis Rat } \\
\text { Sciatic nerve }\end{array}$ & $\begin{array}{l}- \\
- \\
- \\
-\end{array}$ & $\begin{array}{l}\text { Electrophysiological } \\
\text { evaluation ( } 6 \text { weeks) } \\
\text { Histology on nerve and } \\
\text { surrounding tissue (H\&E, } \\
\text { Masson's trichrome) }(1,2,4, \\
6 \text { weeks) } \\
\text { Muscle wet weight }(2,4, \\
6 \text { weeks) } \\
\text { Analysis of the blood-nerve } \\
\text { barrier ( } 2 \text { days) }\end{array}$ & $\begin{array}{l}\text { PLA film has the } \\
\text { potential to prevent } \\
\text { adhesion even after } \\
\text { internal neurolysis, and it } \\
\text { is a useful substitute for } \\
\text { perineurium. }\end{array}$ \\
\hline $\begin{array}{l}\text { Atkins et al., } 2006 \\
\text { [52] }\end{array}$ & Section + suture & $\begin{array}{l}\text { TGF- } \beta 1 \text { and } \\
\text { TGF- } \beta 2\end{array}$ & $\begin{array}{l}\text { Male } \\
\text { Sprague-Dawley } \\
\text { Rat } \\
\text { Sciatic nerve }\end{array}$ & - & $\begin{array}{l}\text { Electrophysiological } \\
\text { evaluation ( } 7 \text { weeks) } \\
\text { Histology on nerve and } \\
\text { surrounding tissue } \\
\text { (Picrosirius staining and } \\
\text { Toluidine Blue for } \\
\text { morphometrical analysis) } \\
\text { (7 weeks) }\end{array}$ & $\begin{array}{l}\text { No differences in the } \\
\text { percentage of collagen } \\
\text { staining area were } \\
\text { observed. Compound } \\
\text { action potential ratios } \\
\text { significantly smaller; } \\
\text { increased number of } \\
\text { myelinated fibers distally } \\
\text { (no differences between } \\
\text { TGF- } \beta 1 \text { and TGF- } \beta 2 \text { ) }\end{array}$ \\
\hline $\begin{array}{l}\text { Gorgulu } \\
\text { et al.,2003 [34] }\end{array}$ & $\begin{array}{l}\text { Neurolysis vs. } \\
\text { scratching vs. } \\
\text { suture vs. } \\
\text { radiation } \\
\text { treatment }\end{array}$ & $\begin{array}{l}\text { Low-dose } \\
\text { radiation therapy } \\
\text { ( } 24 \mathrm{~h} \text { after } \\
\text { surgery) }\end{array}$ & $\begin{array}{l}\text { Male } \\
\text { Sprague-Dawley } \\
\text { Rat } \\
\text { Sciatic nerve }\end{array}$ & $\begin{array}{l}- \\
- \\
-\end{array}$ & $\begin{array}{l}\text { Functional analysis (sciatic } \\
\text { nerve function) (weekly) } \\
\text { Gross evaluation } \\
\text { (Petersen's classification) } \\
\text { (6 weeks) } \\
\text { Histology on nerve and } \\
\text { surrounding tissue } \\
\text { (Masson's trichrome- } \\
\text { fibroblast/fibrocytes count) } \\
\text { (6 weeks) }\end{array}$ & $\begin{array}{l}\text { Significant reduction of } \\
\text { scar tissue in radiation }+ \\
\text { surgery groups. No } \\
\text { increase in scar tissue } \\
\text { formation after radiation } \\
\text { in normal nerves was } \\
\text { observed. }\end{array}$ \\
\hline $\begin{array}{l}\text { Ikeda et al., } 2002 \\
\text { [84] }\end{array}$ & Burning & $\begin{array}{l}\text { Absorbable } \\
\text { oxidized } \\
\text { regenerated } \\
\text { cellulose sheet }\end{array}$ & $\begin{array}{l}\text { White Japanese } \\
\text { Rabbit } \\
\text { Sciatic nerve }\end{array}$ & - & $\begin{array}{l}\text { Electrophysiological } \\
\text { evaluation ( } 6 \text { weeks) } \\
\text { Gross evaluation } \\
\text { (qualitative } \\
\text { evaluation-observation of } \\
\text { Fontana's bands) (6 weeks) } \\
\text { Histology on nerve and } \\
\text { surrounding tissue } \\
\text { (Masson's trichrome) } \\
\text { (6 weeks) }\end{array}$ & $\begin{array}{l}\text { No significant differences } \\
\text { between groups in } \\
\text { electrophysiological } \\
\text { evaluation. High } \\
\text { adhesion between nerve } \\
\text { and surrounding tissue in } \\
\text { the damage group. }\end{array}$ \\
\hline Ip et al., 2000 [15] & Section + suture & $\begin{array}{l}\text { Early } \\
\text { mobilization }\end{array}$ & $\begin{array}{l}\text { Albino Rabbit } \\
\text { Peroneal nerve }\end{array}$ & - & $\begin{array}{l}\text { Biomechanical examination } \\
\text { (stretch test and peel test) } \\
\text { ( } 3 \text { weeks) }\end{array}$ & $\begin{array}{l}\text { No difference in the } \\
\text { biomechanical features of } \\
\text { the adhesions }\end{array}$ \\
\hline
\end{tabular}

A biodegradable polylactide (PLA) honeycomb film [83] and a nerve conduit composed of PLA and poly(e-caprolactone) (PCL) and enriched with hyaluronic acid [57] have been shown to prevent nerve adhesion. Additionally, a novel multilayer membrane made of PLA-based biodegradable polymer (E8002) containing L-ascorbic acid has been demonstrating to reduce scar formation compared to the same membrane without ascorbic acid [82]. Atkins et al. [52] proposed the local administration of neutralizing antibodies to TGF- $\beta 1$ and TGF- $\beta 2$, showing a significant reduction in intraneural scar formation. The use of Ankaferd blood stopper resulted in better healing and better results in the histopathological evaluations [59], and the use of an absorbable oxidized regenerated cellulose sheet showed the prevention of adhesion in a histological study [84]. Finally, low-dose radiation therapy [34] and early mobilization [15] have also been proposed. 


\section{Discussion}

Traction neuropathies are diffused and frequent consequences of injuries or surgical procedures on peripheral nerves [2,7]. Surgeons and researchers have been trying to prevent scar tissue formation, especially by applying antiadhesion devices on the surgical site. Before human implantation, preclinical studies are of crucial importance for assessing the effectiveness of antiadhesion strategies; however, the results reported in the literature are not easily comparable due to the many different methods to induced scar formation as well as quantitatively evaluate the amount of scar tissue and its impact on peripheral nerve regeneration and function. The reason is connected to the absence of a shared, effective, reliable, reproducible, and standardized protocol to induce and test the scar tissue around the peripheral nerves [18,20-22].

The purpose of this review was, therefore, to resume and show the different strategies adopted in the last few years to simulate and evaluate scar tissue formation.

Different kinds of injuries have been proposed by researchers to simulate perineural scar tissue formation: nerve injury, injury to surrounding tissues, global injury by means of chemical or physical agents, and so on. Some authors have designed studies to simulate and evaluate perineural scars [17-22], but these works are incomplete because they have not considered all the aspects of induction and the evaluation methods available. Our review reveals that the widest protocol used to induce scar tissue is represented by section and suture of the nerve. This partially represents what really happens in clinical settings and results in a partial injury. In our opinion, an injury to the perineural tissue should always be associated in clinical settings by means of burning or chemical injury to the nerve. None of the papers have combined this kind of injury. It would, therefore, be very interesting and useful to test the combination of these methods in an experimental model that better mimics the clinic. Furthermore, researchers should consider that a different pattern of scar tissue arises if the nerve is transected or not. Without a nerve section and suture or without a crush injury, no internal scar will form, especially when there has been only a short period between the surgery and the analysis. In this way, no or only minimal impairment of nerve function will be present.

This aspect links to the other main aspect of this review: so many different types of analysis were performed to quantitatively or qualitatively assess the perineural scar and its reduction. We strongly encourage the use of quantitative analysis. Gross evaluation is certainly a fundamental step to macroscopically grading the scar, and the adoption of a numerical grading scheme is necessary to quantify (or semiquantify) the amount of scar tissue. Different grading schemes have been adopted $[13,14,17,23,27,28]$ and most of the authors have used these schemes to score scar tissue during macroscopical inspection. Nevertheless, other authors have described only the presence or absence of scar tissue, and, sometimes, they qualitatively describe the scar tissue by relying on subjective observations.

Microscopical evaluation is usually conducted both on the nerve and scar tissue. Beyond the type of staining used, the quantitative evaluation of the perineural scar is very important. First of all, a staining solution that is able to visualize collagen fibers specifically (such as Masson's Trichrome, Sirius Red) is preferred to the classical H\&E and methylene blue stainings. Quantification of scar tissue is proposed in different ways, but the most adopted is the calculation of the scar tissue formation index (by dividing the mean thickness of the scar tissue by the mean thickness of the nerve tissue) [18,28,30-34,36-41]. Moreover, in these cases, some other authors have used different parameters to quantify (or semiquantify) the scar tissue histologically; sometimes, the structure of scar tissue is only qualitatively described. Some authors have added immunohistochemistry to conventional staining. Very interesting are the attempts to detect scar tissue using antibodies against macrophages and lymphocytes, but it is difficult to obtain a quantification of these findings $[21,37,59]$.

Biomechanical analysis provides another quantitative parameter and consists of measuring the peak force required to pull the nerve from the muscular bed. Different tools have been adopted, and, in general, they consist of applying a continuous force to the nerve until 
the complete detachment of the nerve from the surrounding tissue. This should be the other main method that is useful to demonstrate the strength of the scar tissue in preclinical analysis. In addition, the structure and function of the nerve must be assessed. Quantification of different parameters (number of fibers, axon and fiber diameter, myelin thickness) are important to assess the degree of nerve regeneration and can be related to electrophysiological parameters and functional evaluations, often assessed in studies dealing with peripheral nerve scarring. In our thoughts, morphological and functional impairments correlate more to a direct nerve injury than an experimental perineural scar. Hence, morphometry and electrophysiology should be associated, especially when nerve injury and repair have been performed or when scar neuropathy has lasted for several months.

Different animal models have been proposed, each one with pros and cons. Mice are easy to house and allow quick and easy surgery. The sciatic nerve allows the performance of all the main analyses described above. Otherwise, in this model of nerve repair, functional and electrophysiological tests are more difficult to perform due to the smallness of the animal itself. Conversely, rats allow the testing of both the sciatic nerve and the median nerve; the size of these nerves is feasible for nerve repair and electrodiagnostic tests. Furthermore, functional evaluation on the median nerve can be carried out, especially when a direct nerve injury is performed.

Due to the limitations of the present models, no definitive conclusion can be derived about the efficacy of antiadhesion devices. In most of the experiments, every treated group showed scar reduction according to the evaluation methods. The most employed antiadhesion devices were polysaccharide-based and collagen-based ones. Their effectiveness was, in most cases, well known in spine surgery, tendon surgery, or abdominal surgery. It is similar for biological barriers: vein wrapping has been described to protect nerve sutures in the past $[25,85]$, with good clinical outcomes. Fat grafts were previously adopted in spine surgery with controversial results; in peripheral nerves, it seems to be effective, and promising results were obtained with Coleman's lipoaspirate [70]. From our review, it has emerged that the application of amniotic membrane can be promising, considering the increased chance of tissue storage $[14,39,40]$. This requirement can also be considered a limit for this technique. Drugs and other devices can also be promising, but currently, no clinical experience exists.

This preclinical literature review suggests that we reconsider the whole argument of traction neuropathies. This pathology was classified by Millesi [86] in intra- and extraneural scar, but more extensive classification would be effective to better understand the correct treatment. Perineural scarring arises after closed trauma and nerve decompression or can be associated with a repaired nerve. The intraneural involvement is different in each case, and different approaches should be considered. When an intraneural scar is present, it should be treated as a neuroma in continuity. In contrast, when the perineural scar is the main concern, other procedures should be performed. According to our clinical practice and the results obtained in these experimental models, vein wrapping can be a feasible procedure to prevent intraneural and epineural adhesion after nerve suture. Otherwise, in a secondary peripheral nerve decompression, the application of an antiadhesion device, either in the form of gel or film composed of polysaccharide or collagen, could be adequate as well as autologous lipoaspirate, local adipose flap, synovial flap, or amniotic membrane wrapping. The choice, at state of the art, is up to the individual surgeon's experience and availability since no clear evidence exists.

To better understand traction neuropathies, a more extensive classification should be designed by considering the extension of the scar, the amount of fibrous tissue (both preand intraoperative by means of a quantitative scale, as proposed by Petersen [27]), and previous surgery on the nerve (suture, traction injury, nerve decompression). On these, prognostic criteria would be found and a more fitted treatment protocol developed.

In our experience and considering the literature, to completely evaluate scar tissue formation around a nerve, we require a scored macroscopical analysis, a quantitative microscopical analysis conducted with a staining solution that is able to collagen fibers 
visualize specifically (such as Masson's Trichrome, Sirus Red) in order to clearly measure the thickness and extension of scar tissue, and a computed biomechanical analysis with the appropriate microinstruments. The structure, organization, and function of the nerve should also accompany scar tissue data, but these are not always mandatory since they depend on the type of nerve injury induced.

Finally, this review reports on the different antiadhesion devices that have been experimentally tested so far. Due to the high variability of scar induction and evaluation methods described, it is not possible to compare the results obtained in terms of scar reduction and efficacy of the antiadhesion devices employed.

Author Contributions: A.C., G.R. and P.T. organized the manuscript. A.C., G.R., B.E.F., S.O. and S.R. wrote different sections of the manuscript. All authors contributed to manuscript revision and approved the submitted version of the manuscript.

Funding: This research received no external funding.

Informed Consent Statement: Not applicable.

Conflicts of Interest: The authors declare no conflict of interest.

\section{References}

1. Tos, P.; Crosio, A.; Pugliese, P.; Adani, R.; Toia, F.; Artiaco, S. Topic: Peripheral Nerve Repair and Regeneration Painful scar neuropathy: Principles of diagnosis and treatment. Plast. Aesthet. Res. 2015, 2. Available online: www.parjournal.net (accessed on 18 May 2020). [CrossRef]

2. Jones, N.F.; Ahn, H.C.; Eo, S. Revision surgery for persistent and recurrent carpal tunnel syndrome and for failed carpal tunnel release. Plast. Reconstr. Surg. 2012, 129, 683-692. [CrossRef]

3. Siemionow, M.; Brzezicki, G. Chapter 8 Current Techniques and Concepts in Peripheral Nerve Repair. Int. Rev. Neurobiol. 2009, 87, 141-172. [CrossRef] [PubMed]

4. Wiberg, M.; Terenghi, G. Will it be possible to produce peripheral nerves? Surg. Technol. Int. 2003, 11, 303-310.

5. Geuna, S. Appreciating the difference between design-based and model-based sampling strategies in quantitative morphology of the nervous system. J. Comp. Neurol. 2000, 427, 333-339. [CrossRef]

6. Siemionow, M.; Mendiola, A. Methods of assessment of cortical plasticity in patients following amputation, replantation, and composite tissue allograft transplantation. Ann. Plast. Surg. 2010, 65, 344-348. [CrossRef] [PubMed]

7. Amadio, P.C. Interventions for recurrent/persistent carpal tunnel syndrome after carpal tunnel release. J. Hand Surg. 2009, 34, 1320-1322. [CrossRef] [PubMed]

8. Sunderland, S. Nerves and Nerve Injuries, 2nd ed.; Churchill Livingstone: Edinburgh, UK, 1978.

9. Wang, M.L.; Rivlin, M.; Graham, J.G.; Beredjiklian, P.K. Peripheral nerve injury, scarring, and recovery. Connect. Tissue Res. 2019, 60, 3-9. [CrossRef]

10. Smit, X.; van Neck, J.W.; Afoke, A.; Hovius, S.E.R. Reduction of neural adhesions by biodegradable autocrosslinked hyaluronic acid gel after injury of peripheral nerves: An experimental study. J. Neurosurg. 2004, 101, 648-652. [CrossRef] [PubMed]

11. Ronchi, G.; Morano, M.; Fregnan, F.; Pugliese, P.; Crosio, A.; Tos, P.; Geuna, S.; Haastert-Talini, K.; Gambarotta, G. The median nerve injury model in pre-clinical research-A critical review on benefits and limitations. Front. Cell. Neurosci. 2019, 13. [CrossRef]

12. Diogo, C.C.; Camassa, J.A.; Pereira, J.E.; da Costa, L.M.; Filipe, V.; Couto, P.A.; Geuna, S.; Maurício, A.C.; Varejão, A.S. The use of sheep as a model for studying peripheral nerve regeneration following nerve injury: Review of the literature. Neurol. Res. 2017, 39, 926-939. [CrossRef]

13. Isla, A.; Martinez, J.; Perez-Lopez, C.; Conde, C.P.; Morales, C.; Budke, M. A reservable antiadhesion barrier gel reduces the perineural adhesions in rats after anastomosis/Comment. J. Neurosurg. Sci. 2003, 47, 195-199. Available online: http: / / search.proquest.com/openview /298ef0c6b31f2fb391e0685f5ab5c636/1?pq-origsite=gscholar\&cbl=49236 (accessed on 19 May 2020). [PubMed]

14. Kim, S.S.; Sohn, S.K.; Lee, K.Y.; Lee, M.J.; Roh, M.S.; Kim, C.H. Use of human amniotic membrane wrap in reducing perineural adhesions in a rabbit model of ulnar nerve neurorrhaphy. J. Hand Surg. Eur. Vol. 2010, 35, 214-219. [CrossRef]

15. Ip, W.Y.; Shibata, T.; Tang, F.H.; Mak, A.F.T.; Chow, S.P. Adhesion formation after nerve repair: An experimental study of early protected mobilization in the rabbit. J. Hand Surg. 2000, 25, 582-584. [CrossRef] [PubMed]

16. Colonna, M.R.; Fazio, A.; Costa, A.L.; Galletti, F.; lo Giudice, R.; Galletti, B.; Galletti, C.; lo Giudice, G.; Orabona, G.D.; Papalia, I.; et al. The Use of a Hypoallergenic Dermal Matrix for Wrapping in Peripheral Nerve Lesions Regeneration: Functional and Quantitative Morphological Analysis in an Experimental Animal Model. BioMed Res. Int. 2019, 2019. [CrossRef] [PubMed]

17. Abe, Y.; Doi, K.; Kawai, S. An experimental model of peripheral nerve adhesion in rabbits. Br. J. Plast. Surg. 2005, 58, 533-540. [CrossRef] [PubMed]

18. Zanjani, L.O.; Firouzi, M.; Nabian, M.-H.; Nategh, M.; Rahimi-Movaghar, V.; Kamrani, R.S. Comparison and Evaluation of Current Animal Models for Perineural Scar Formation in Rat. Iran. J. Basic Med. Sci. 2013, 16, 890. 
19. Okuhara, Y.; Shinomiya, R.; Peng, F.; Kamei, N.; Kurashige, T.; Yokota, K.; Ochi, M. Direct effect of radiation on the peripheral nerve in a rat model. J. Plast. Surg. Hand Surg. 2014, 48, 276-280. [CrossRef] [PubMed]

20. Crosio, A.; Valdatta, L.; Cherubino, M.; Izzo, M.; Pellegatta, I.; Pascal, D.; Geuna, S.; Tos, P. A simple and reliable method to perform biomechanical evaluation of postoperative nerve adhesions. J. Neurosci. Methods 2014, 233, 73-77. [CrossRef]

21. Lemke, A.; Penzenstadler, C.; Ferguson, J.; Lidinsky, D.; Hopf, R.; Bradl, M.; Redl, H.; Wolbank, S.; Hausner, T. A novel experimental rat model of peripheral nerve scarring that reliably mimics post-surgical complications and recurring adhesions. Dis. Models Mech. 2017, 10, 1015-1025. [CrossRef]

22. Zuijdendorp, H.; Smit, X.; Blok, J.; Caruelle, J.; Barritault, D.; Hovius, S.; van Neck, J. Significant reduction in neural adhesions after administration of the regenerating agent OTR4120, a synthetic glycosaminoglycan mimetic, after peripheral nerve injury. J. Neurosurg. 2008, 109, 967-973. Available online: https://thejns.org/view/journals/j-neurosurg/109/5/article-p967.xml (accessed on 19 May 2020). [CrossRef] [PubMed]

23. Palatinsky, E.A.; Maier, K.H.; Touhalisky, D.K.; Mock, J.L.; Hingson, M.T.; Coker, G.T. ADCON ${ }^{\circledR}-T / N$ reduces in vivo perineural adhesions in a rat sciatic nerve reoperation model. J. Hand Surg. Eur. Vol. 1997, 22, 331-335. [CrossRef]

24. Ikeda, K.; Yamauchi, D.; Osamura, N.; Hagiwara, N.; Tomita, K. Hyaluronic acid prevents peripheral nerve adhesion. Br. J. Plast. Surg. 2003, 56, 342-347. [CrossRef]

25. Xu, J.; Varitimidis, S.E.; Fisher, K.J.; Tomaino, M.M.; Sotereanos, D.G. The effect of wrapping scarred nerves with autogenous vein graft to treat recurrent chronic nerve compression. J. Hand Surg. 2000, 25, 93-103. [CrossRef] [PubMed]

26. Animals NRC (US). C on R and A of P in L. Recognition and Alleviation of Pain in Laboratory Animals; National Academies Press: Washington, DC, USA, 2010. [CrossRef]

27. Petersen, J.; Russell, L.; Andrus, K.; MacKinnon, M.; Silver, J.; Kliot, M. Reduction of extraneural scarring by ADCON-T/N after surgical intervention. Neurosurgery 1996, 38, 976-984. [CrossRef] [PubMed]

28. Dam-Hieu, P.; Lacroix, C.; Said, G.; Devanz, P.; Liu, S.; Tadie, M. Reduction of postoperative perineural adhesions by hyaloglide gel: An experimental study in the rat sciatic nerve. Neurosurgery 2005, 56 (Suppl. S4). [CrossRef] [PubMed]

29. Junqueira, L.C.U.; Bignolas, G.; Brentani, R.R. Picrosirius staining plus polarization microscopy, a specific method for collagen detection in tissue sections. Histochem. J. 1979, 11, 447-455. [CrossRef]

30. Albayrak, B.S.; Ismailoglu, O.; Ilbay, K.; Yaka, U.; Tanriover, G.; Gorgulu, A.; Demir, N. Doxorubicin for prevention of epineurial fibrosis in a rat sciatic nerve model: Outcome based on gross postsurgical, histopathological, and ultrastructural findingsLaboratory investigation. J. Neurosurg. Spine 2010, 12, 327-333. [CrossRef]

31. Özay, R.; Bekar, A.; Kocaeli, H.; Karli, N.; Filiz, G.; Ulus, I.H. Citicoline improves functional recovery, promotes nerve regeneration, and reduces postoperative scarring after peripheral nerve surgery in rats. Surg. Neurol. 2007, 68, 615-622. [CrossRef]

32. Ilbay, K.; Etus, V.; Yildiz, K.; Ilbay, G.; Ceylan, S. Topical application of mitomycin C prevents epineural scar formation in rats. Neurosurg. Rev. 2005, 28, 148-153. [CrossRef]

33. Görgülü, A.; Imer, M.; Şimşek, O.; Sencer, A.; Kutlu, K.; Çobanoğlu, S. The effect of aprotinin on extraneural scarring in peripheral nerve surgery: An experimental study. Acta Neurochir. 1998, 140, 1303-1307. [CrossRef] [PubMed]

34. Görgülü, A.; Uzal, C.; Doğanay, L.; Imer, M.; Eliuz, K.; Çobanoğlu, S.; Loeffler, J.S.; Gerszten, P.C.; Kondziolka, D.; Kline, D.G. The effect of low-dose external beam radiation on extraneural scarring after peripheral nerve surgery in rats. Neurosurgery 2003, 53, 1389-1396. [CrossRef] [PubMed]

35. Özgenel, G.Y. Effects of hyaluronic acid on peripheral nerve scarring and regeneration in rats. Microsurgery 2003, $23,575-581$. [CrossRef] [PubMed]

36. Mekaj, A.Y.; Manxhuka-Kerliu, S.; Morina, A.A.; Duci, S.B.; Shahini, L.; Mekaj, Y.H. Effects of hyaluronic acid and tacrolimus on the prevention of perineural scar formation and on nerve regeneration after sciatic nerve repair in a rabbit model. Eur. J. Trauma Emerg. Surg. 2017, 43, 497-504. [CrossRef]

37. Marcol, W.; Larysz-Brysz, M.; Kucharska, M.; Niekraszewicz, A.; Slusarczyk, W.; Kotulska, K.; Wlaszczuk, P.; Wlaszczuk, A.; Jedrzejowska-Szypulka, H.; Lewin-Kowalik, J. Reduction of post-traumatic neuroma and epineural scar formation in rat sciatic nerve by application of microcrystallic chitosan. Microsurgery 2011, 31, 642-649. [CrossRef]

38. Park, J.S.; Lee, J.H.; Han, C.S.; Chung, D.W.; Kim, G.Y. Effect of hyaluronic acid-carboxymethylcellulose solution on perineural scar formation after sciatic nerve repair in rats. Clin. Orthop. Surg. 2011, 3, 315-324. [CrossRef]

39. Meng, H.; Li, M.; You, F.; Du, J.; Luo, Z. Assessment of processed human amniotic membrane as a protective barrier in rat model of sciatic nerve injury. Neurosci. Lett. 2011, 496, 48-53. [CrossRef]

40. Özgenel, G.Y.; Fílíz, G. Combined application of human amniotic membrane wrapping and hyaluronic acid injection in epineurectomized rat sciatic nerve. J. Reconstr. Microsurg. 2004, 20, 153-157. [CrossRef]

41. Vural, E.; Yilmaz, M.; Ilbay, K.; Ilbay, G. Prevention of epineural fibrosis in rats by local administration of mitomycin C or daunorubicin. Turk. Neurosurg. 2016, 26, 291-296. [CrossRef]

42. Sakurai, M.; Miyasaka, Y. Neural fibrosis and the effect of neurolysis. J. Bone Joint Surg. Ser. B 1986, 68, 483-488. [CrossRef]

43. He, Y.; Revel, M.; Loty, B. A quantitative model of post-laminectomy scar formation: Effects of a nonsteroidal anti-inflammatory drug. Spine 1995, 20, 557-563. [CrossRef]

44. Hinton, J.L.; Warejcka, D.J.; Mei, Y.; McLendon, R.E.; Laurencin, C.; Lucas, P.A.; Robinson, J.S. Inhibition of epidural scar formation after lumbar laminectomy in the rat. Spine 1995, 20, 564-570. [CrossRef] 
45. Ornelas, L.; Padilla, L.; di Silvio, M.; Schalch, P.; Esperante, S.; Infante, R.L.; Bustamante, J.C.; Avalos, P.; Varela, D.; López, M. Fibrin glue: An alternative technique for nerve coaptation-Part II. Nerve regeneration and histomorphometric assessment. J. Reconstr. Microsurg. 2006, 22, 123-128. [CrossRef]

46. Baltu, Y.; Uzun, H.; Özgenel, G.Y. The reduction of extraneural scarring with buccal mucosa graft wrapping around the sciatic nerve: An experimental study in a rat model. J. Plast. Surg. Hand Surg. 2017, 51, 259-263. [CrossRef]

47. Mathieu, L.; Adam, C.; Legagneux, J.; Bruneval, P.; Masmejean, E. Reduction of neural scarring after peripheral nerve suture: An experimental study about collagen membrane and autologous vein wrapping. Chir. Main 2012, 31, 311-317. [CrossRef] [PubMed]

48. Li, R.; Liu, H.; Huang, H.; Bi, W.; Yan, R.; Tan, X.; Wen, W.; Wang, C.; Song, W.; Zhang, Y.; et al. Chitosan conduit combined with hyaluronic acid prevent sciatic nerve scar in a rat model of peripheral nerve crush injury. Mol. Med. Rep. 2018, 17, 4360-4368. [CrossRef] [PubMed]

49. Adanali, G.; Verdi, M.; Tuncel, A.; Erdogan, B.; Kargi, E. Effects of hyaluronic acid-carboxymethylcellulose membrane on extraneural adhesion formation and peripheral nerve regeneration. J. Reconstr. Microsurg. 2003, 19, 29-35. [CrossRef] [PubMed]

50. Ngeow, W.C.; Atkins, S.; Morgan, C.R.; Metcalfe, A.D.; Boissonade, F.M.; Loescher, A.R.; Robinson, P.P. A comparison between the effects of three potential scar-reducing agents applied at a site of sciatic nerve repair. Neuroscience 2011, 181, 271-277. [CrossRef]

51. Ngeow, W.C.; Atkins, S.; Morgan, C.R.; Metcalfe, A.D.; Boissonade, F.M.; Loescher, A.R.; Robinson, P.P. The effect of Mannose-6Phosphate on recovery after sciatic nerve repair. Brain Res. 2011, 1394, 40-48. [CrossRef]

52. Atkins, S.; Smith, K.G.; Loescher, A.R.; Boissonade, F.M.; Ferguson, M.W.J.; Robinson, P.P. The effect of antibodies to TGF- $\beta 1$ and TGF- $\beta 2$ at a site of sciatic nerve repair. J. Peripher. Nerv. Syst. 2006, 11, 286-293. [CrossRef]

53. Atkins, S.; Loescher, A.R.; Boissonade, F.M.; Smith, K.G.; Occleston, N.; O'Kane, S.; Ferguson, M.W.J.; Robinson, P.P. Interleukin-10 reduces scarring and enhances regeneration at a site of sciatic nerve repair. J. Peripher. Nerv. Syst. 2007, 12, 269-276. [CrossRef]

54. Geuna, S. The revolution of counting "tops": Two decades of the disector principle in morphological research. Microsc. Res. Tech. 2005, 66, 270-274. [CrossRef]

55. Brown, R.E.; Erdmann, D.; Lyons, S.F.; Suchy, H. The use of cultured Schwann cells in nerve repair in a rabbit hind-limb model. J. Reconstr. Microsurg. 1996, 12, 149-152. [CrossRef]

56. Aslan, E.; Kocaeli, H.; Bekar, A.; Tolunay, Ş.; Ulus, I.H. CDP-choline and its endogenous metabolites, cytidine and choline, promote the nerve regeneration and improve the functional recovery of injured rat sciatic nerves. Neurol. Res. 2011, 33, 766-773. [CrossRef]

57. Shintani, K.; Uemura, T.; Takamatsu, K.; Yokoi, T.; Onode, E.; Okada, M.; Nakamura, H. Protective effect of biodegradable nerve conduit against peripheral nerve adhesion after neurolysis. J. Neurosurg. 2018, 129, 815-824. [CrossRef]

58. Que, J.; Cao, Q.; Sui, T.; Du, S.; Kong, D.; Cao, X. Effect of FK506 in reducing scar formation by inducing fibroblast apoptosis after sciatic nerve injury in rats. Cell Death Dis. 2013, 4. [CrossRef]

59. Servet, E.; Bekler, H.; Kilinçoğlu, V.; Özler, T.; Özkut, A. Effect of bleeding on nerve regeneration and epineural scar formation in rat sciatic nerves: An experimental study. Acta Orthop. Traumatol. Turc. 2016, 50, 234-241. [CrossRef] [PubMed]

60. Xue, J.W.; Jiao, J.B.; Liu, X.F.; Jiang, Y.T.; Yang, G.; Li, C.Y.; Yin, W.T.; Ling, L. Inhibition of peripheral nerve scarring by calcium antagonists, also known as calcium channel blockers. Artif. Organs 2016, 40, 514-520. [CrossRef] [PubMed]

61. Zhu, D.; Tapadia, M.D.; Palispis, W.; Luu, M.; Wang, W.; Gupta, R. Attenuation of robust glial scar formation facilitates functional recovery in animal models of chronic nerve compression injury. J. Bone Joint Surg. Am. Vol. 2017, 99, e132. [CrossRef] [PubMed]

62. Murakami, K.; Kuniyoshi, K.; Iwakura, N.; Matsuura, Y.; Suzuki, T.; Takahashi, K.; Ohtori, S. Vein wrapping for chronic nerve constriction injury in a rat model: Study showing increases in VEGF and HGF production and prevention of pain-associated behaviors and nerve damage. J. Bone Joint Surg. Am. Vol. 2014, 96, 859-867. [CrossRef]

63. Bain, J.R.; Mackinnon, S.E.; Hunter, D.A. Functional evaluation of complete sciatic, peroneal, and posterior tibial nerve lesions in the rat. Plast. Reconstr. Surg. 1989, 83, 129-136. [CrossRef] [PubMed]

64. Nakamura, S.; Atsuta, Y. The effects of experimental neurolysis on ectopic firing in a rat chronic constriction nerve injury model. J. Hand Surg. 2006, 31, 35-39. [CrossRef] [PubMed]

65. Stößel, M.; Rehra, L.; Haastert-Talini, K. Reflex-based grasping, skilled forelimb reaching, and electrodiagnostic evaluation for comprehensive analysis of functional recovery-The 7-mm rat median nerve gap repair model revisited. Brain Behav. 2017, 7. [CrossRef]

66. Dumanian, G.A.; McClinton, M.; Brushart, T.M. The effects of free fat grafts on the stiffness of the rat sciatic nerve and perineural scar. J. Hand Surg. 1999, 24, 30-36. [CrossRef] [PubMed]

67. Yamamoto, M.; Endo, N.; Ito, M.; Okui, N.; Koh, S.; Kaneko, H.; Hirata, H. Novel polysaccharide-derived hydrogel prevents perineural adhesions in a rat model of sciatic nerve adhesion. J. Orthop. Res. 2010, 28, 284-288. [CrossRef] [PubMed]

68. Urano, H.; Iwatsuki, K.; Yamamoto, M.; Ohnisi, T.; Kurimoto, S.; Endo, N.; Hirata, H. Novel anti-adhesive CMC-PE hydrogel significantly enhanced morphological and physiological recovery after surgical decompression in an animal model of entrapment neuropathy. PLoS ONE 2016, 11, e0164572. [CrossRef] [PubMed]

69. Ohsumi, H.; Hirata, H.; Nagakura, T.; Tsujii, M.; Sugimoio, T.; Miyamoto, K.; Horiuchi, T.; Nagao, M.; Nakashima, T.; Uchida, A. Enhancement of perineurial repair and inhibition of nerve adhesion by viscous injectable pure alginate sol. Plast. Reconstr. Surg. 2005, 116, 823-830. [CrossRef]

70. Cherubino, M.; Pellegatta, I.; Crosio, A.; Valdatta, L.; Geuna, S.; Gornati, R.; Tos, P. Use of human fat grafting in the prevention of perineural adherence: Experimental study in athymic mouse. PLoS ONE 2017, 12, e0176393. [CrossRef] 
71. Tos, P.; Crosio, A.; Pellegatta, I.; Valdatta, L.; Pascal, D.; Geuna, S.; Cherubino, M. Efficacy of anti-adhesion gel of carboxymethylcellulose with polyethylene oxide on peripheral nerve: Experimental results on a mouse model. Muscle Nerve 2016, 53, 304-309. [CrossRef]

72. Magill, C.K.; Tuffaha, S.H.; Yee, A.; Luciano, J.P.; Hunter, D.A.; Mackinnon, S.E.; Borschel, G.H. The short- and long-term effects of Seprafilm ${ }^{\circledR}$ on peripheral nerves: A histological and functional study. J. Reconstr. Microsurg. 2009, 25, 345-354. [CrossRef]

73. Hachinota, A.; Tada, K.; Yamamoto, D.; Nakajima, T.; Nakada, M.; Tsuchiya, H. Preventive effect of alginate gel formulation on perineural adhesion. J. Hand Surg. Asian Pac. Vol. 2020, 25, 164-171. Available online: https:/ / pubmed-ncbi-nlm-nih-gov. bibliopass.unito.it/32312202/ (accessed on 14 December 2020). [CrossRef] [PubMed]

74. Hernández-Cortés, P.; Peregrina, M.; Aneiros-Fernández, J.; Tassi, M.; Pajares-López, M.; Toledo, M.; O’Valle, F. Oxidized regenerated cellulose does not prevent the formation of experimental postoperative perineural fibrosis assessed by digital analysis. Histol. Histopathol. 2010, 25, 741-747. [CrossRef] [PubMed]

75. Kim, P.D.; Hayes, A.; Amin, F.; Akelina, Y.; Hays, A.P.; Rosenwasser, M.P. Collagen nerve protector in rat sciatic nerve repair: A morphometric and histological analysis. Microsurgery 2010, 30, 392-396. [CrossRef] [PubMed]

76. Lee, J.Y.; Parisi, T.J.; Friedrich, P.F.; Bishop, A.T.; Shin, A.Y. Does the addition of a nerve wrap to a motor nerve repair affect motor outcomes? Microsurgery 2014, 34, 562-567. [CrossRef]

77. Jay, R.M.; Huish, J.P.; Wray, J.H. Amniotic membrane in clinical medicine: History, current status, and future use. In Extracellular Matrix-Derived Implants in Clinical Medicine; Elsevier Inc.: Amsterdam, The Netherlands, 2016; pp. 151-176. [CrossRef]

78. Kaplan, T.; Kafa, I.M.; Cansev, M.; Bekar, A.; Karli, N.; Taskapilioglu, M.O.; Kanar, F. Investigation of the dose-dependency of citicoline effects on nerve regeneration and functional recovery in a rat model of sciatic nerve injury. Turk. Neurosurg. 2014, 24, 54-62. [CrossRef] [PubMed]

79. Shahraki, M.; Mohammadi, R.; Najafpour, A. Influence of tacrolimus (FK506) on nerve regeneration using allografts: A rat sciatic nerve model. J. Oral Maxillofac. Surg. 2015, 73, 1438.e1-1438.e9. [CrossRef]

80. Davis, B.; Hilgart, D.; Erickson, S.; Labroo, P.; Burton, J.; Sant, H.; Shea, J.; Gale, B.; Agarwal, J. Local FK506 delivery at the direct nerve repair site improves nerve regeneration. Muscle Nerve 2019, 60, 613-620. [CrossRef]

81. Stocco, E.; Barbon, S.; Macchi, V.; Tiengo, C.; Petrelli, L.; Rambaldo, A.; Borean, A.; Capelli, S.; Filippi, A.; Romanato, F.; et al. New bioresorbable wraps based on oxidized polyvinyl alcohol and leukocyte-fibrin-platelet membrane to support peripheral nerve neurorrhaphy: Preclinical comparison versus NeuraWrap. Sci. Rep. 2019, 9. Available online: https:/ / pubmed-ncbi-nlm-nih-gov. bibliopass.unito.it/31748615/ (accessed on 14 December 2020). [CrossRef]

82. Kikuchi, K.; Setoyama, K.; Takada, S.; Otsuka, S.; Nakanishi, K.; Norimatsu, K.; Tani, A.; Sakakima, H.; Kawahara, K.I.; Hosokawa, K.; et al. E8002 inhibits peripheral nerve adhesion by enhancing fibrinolysis of l-ascorbic acid in a rat sciatic nerve model. Int. J. Mol. Sci. 2020, 21, 3972. Available online: https:/ / pubmed-ncbi-nlm-nih-gov.bibliopass.unito.it/32492845/ (accessed on 14 December 2020). [CrossRef]

83. Okui, N.; Yamamoto, M.; Fukuhira, Y.; Kaneko, H.; Hirata, H. Artificial perineurium to enhance nerve recovery from damage after neurolysis. Muscle Nerve 2010, 42, 570-575. [CrossRef]

84. Ikeda, K.; Yamauchi, D.; Tomita, K. Preliminary study for prevention of neural adhesion using an absorbable oxidised regenerated cellulose sheet. Hand Surg. 2002, 7, 11-14. [CrossRef] [PubMed]

85. Masear, V.R.; Tullos, J.; St Mary, E.; Mayer, R. Venous wrapping of nerves to prevent to prevent scarring. J. Hand Surg. 1990, 15, 817-818. Available online: https:/ / ci.nii.ac.jp/naid/10026286269/ (accessed on 23 March 2021).

86. Millesi, H.; Zoch, G.; Reihsner, R. Mechanical properties of peripheral nerves. Clin. Orthop. Relat. Res. 1995, 314, 76-83. Available online: https:/ / europepmc.org/article/med/7634654 (accessed on 23 March 2021). [CrossRef] 